\title{
Gevrey Wellposedness of the Cauchy Problem for the Hyperbolic Equations of Third Order with Coefficients Depending Only on Time
}

\author{
Tamotu KinOSHITA*
}

\begin{abstract}
We shall show the Gevrey wellposedness of the Cauchy problem for the hyperbolic equations of third order with coefficients depending only on tıme. For the proof we use the suitable energy of the third order equations.
\end{abstract}

\section{§1. Introduction}

For the hyperbolic equations of second order, F. Colombini, E. De Giorgi, E. Jannelli and S. Spagnolo got the results concerned with the relation between the Gevrey wellposedness and the regularity of the coefficients (see [2], [3] and see also [4], [6], [10]). In this paper we shall extend their results to the hyperbolic equations of third order.

We shall first consider the equation of third order in $[0, T] \times \mathbb{R}_{x}^{n}$

$$
\left\{\begin{array}{l}
u_{t t t}+\sum_{t=1}^{n} a_{\imath}(t) u_{t t x_{t}}+\sum_{i, j=1}^{n} b_{\imath \jmath}(t) u_{t x_{\iota} x}=0 \\
u_{u}(0, x)=u_{0}(x), \quad u_{t}(0, x)=u_{1}(x), \quad u_{t t}(0, x)=u_{2}(x),
\end{array}\right.
$$

where $a_{\imath}(t)$ and $b_{\imath}(t)$ are the real coefficients satisfying

$$
\begin{aligned}
& \text { (2) } \quad \begin{array}{l}
a_{\imath}(t) \in C^{\frac{k+\alpha}{2}}([0, T]) \quad \text { with } k=0 \text { or } 1 \\
\left|a_{\imath}(t)\right| \in C^{\frac{k+\alpha}{2}}([0, T]) \quad k \text { integer } \geq 2
\end{array} \text { and } 0 \leq \alpha \leq 1 \quad(i=1, \cdots, n), \\
& \text { (3) } b_{\imath j}(t) \in C^{k+\alpha}([0, T]) \quad \text { with } k \text { integer } \geq 0 \text { and } 0 \leq \alpha \leq 1 \quad(i, j=1, \cdots, n) .
\end{aligned}
$$

Communicated by T. Kawa1, 6 October, 1997

1991 Mathematics Subject Classifications: 35B65, 35L30

* Institute of Mathematics. University of Tsukuba, Ibaraki 305, Japan. 
Now we assume the restricted type of the weakly hyperbolic condition for the third order equation (1)

$$
\sum_{i, j=1}^{n} b_{i j}(t) \xi_{i} \xi_{j} \leq 0 \text { for }{ }^{\forall} t \in[0, T], \quad{ }^{\forall} \xi \in \mathbb{R}_{\xi}^{n} .
$$

In general, the suitable weakly hyperbolic condition for the equation (1) is

$$
\left(\sum_{i=1}^{n} a_{i}(t) \xi_{i}\right)^{2}-4 \sum_{i, j=1}^{n} b_{\imath}(t) \xi_{i} \xi_{j} \geq 0 \quad \text { for }{ }^{\forall} t \in[0, T], \quad{ }^{\forall} \xi \in \mathbb{R}_{\xi}^{n} .
$$

We can easily see that the condition (4) is stronger than the condition (5). The third order equations have 3 real characteristic roots $\lambda_{1}(t, \xi), \lambda_{2}(t, \xi), \lambda_{3}(t, \xi)$ such that $\lambda_{1}(t, \xi) \leq \lambda_{2}(t, \xi) \leq \lambda_{3}(t, \xi)$ for ${ }^{\forall} t \in[0, T],{ }^{\forall} \xi \in \mathbb{R}_{\xi}^{n}$. Thanks to the condition (4), we find that the characteristic roots of the equation (1) satisfy $\lambda_{1}(t, \xi) \leq 0, \lambda_{2}(t, \xi) \equiv 0, \lambda_{3}(t, \xi) \geq 0$. This fact gives many benefits in the treatment of the hyperbolic equations of third order. In this paper we don't use the condition (5). Actually changing the characteristic direction, any type of weakly hyperbolic equations of third order can be reduced to the equation (1) under the condition (4).

Then we can prove the following theorem (concerning the difinitions of the spaces of functions, see the end of $\S 1$ ).

Theorem 1 . Let $T>0, \mu_{0}>0$. The coefficients satisfy (2), (3) and (4). Then for any $u_{0}, u_{1}$ and $u_{2} \in \gamma^{s}$, the Cauchy problem (1) has a unique (global) solution $u \in C^{3}\left([0, T], \gamma^{s}\right)$, provided

$$
1 \leq s<1+\frac{k+\alpha}{2}
$$

Moreover when $u_{0}, u_{1}$ and $u_{2} \in \gamma_{0}^{s}(s>1)$, there exist the constant $\nu>0$ and the positive function $\mu(t)$ satisfying $\mu(0)=\mu_{0}$, such that for ${ }^{\forall} \xi \in \mathbb{R}_{\xi}^{n}$

$$
e^{\mu(t)\langle\xi\rangle_{\nu}^{\frac{1}{\delta}}}\left(\langle\xi\rangle_{\nu}^{\frac{4}{2+k+\alpha}}|\widehat{u}|+\langle\xi\rangle_{\nu}^{\frac{2}{2+k+\alpha}}\left|\widehat{u}_{t}\right|+\left|\widehat{u}_{t}\right|\right) \leq{ }^{\exists} C e^{\mu_{0}\langle\xi\rangle_{\nu}^{\frac{1}{s}}}\left(\langle\xi\rangle_{\nu}^{2}\left|\widehat{u}_{0}\right|+\langle\xi\rangle_{\nu}\left|\widehat{u}_{1}\right|+\left|\widehat{u}_{2}\right|\right) .
$$

Remark 1. If $k=\alpha=0$, (6) doesn't make sense. However whenever the coefficients $a_{i}(t), b_{i},(t)$ belong to $C^{0}([0, T])$, or even to $L^{1}([0, T])$, the Cauthy problem (1) is wellposed in $\gamma^{1}$ (see [7] and see also [2]).

Remark 2. If one replaced the weakly hyperbolic condition (4) by the condition (5), the same regularity as the coefficients $b_{\imath}(t)$ would be needed for the coefficients $a_{\imath}(t)$, i.e., $a_{\imath}(t)$ or $\left|a_{\imath}(t)\right| \in C^{k+\alpha}([0, T])$.

Remark 3. Precisely the positive function $\mu(t)$ is a strictly decreasing function. Therefore $\mu(T)$ is less than $\mu_{0}(=\mu(0))$. However if we take large 
enough $\nu>0, \mu(T)$ can be chosen arbitrarily close to $\mu_{0}$.

With a different method, Y. Ohya and S. Tarama got more general results for the weakly hyperbolic equations of higher order (see [11] and see also $[14]$ ). They assume that all the coefficients of the highest order terms belong to the same Hölder class with respect to time. For the third order equation (1) whose coefficients satisfy (2), (3) when $k=0$, we relax the regularity of the coefficients $a_{i}(t)$ from $C^{\alpha}$ to $C^{\frac{\alpha}{2}}$. Moreover according to their result, in order that the Cauthy problem for the weakly hyperbolic equations of third order is well-posed in $\gamma^{s}$, it is necessary that the Gevrey exponent $s$ satisfies

$$
1 \leq s<1+\frac{\alpha}{r} \quad(\text { the multiplicity } r=3) \text {. }
$$

The multiplicity of the characteristic roots for the equation (1), is also 3 , but the range (6) when $k=0$, is wider than the range (8). We know that the range (6) for the third order equation (1) coincides the range for the second order equations (see [3]). This improvement is due to the fact that one of the characteristic roots is identically equal to 0 and the regularities of the other two characteristic roots become more smooth.

We shall next consider the strictly hyperbolic case. Instead of the weakly hyperbolic condition (4), we assume the restricted type of the strictly hyperbolic condition for the third order equation (1)

$$
\sum_{i, j=1}^{n} b_{i j}(t) \xi_{i} \xi_{j}<-\delta|\xi|^{2}\left({ }^{\exists} \delta>0\right) \quad \text { for }{ }^{\forall} t \in[0 . T], \quad{ }^{\forall} \xi \in \mathbb{R}_{\xi}^{n} .
$$

In this case we don't need to consider the smooth coefficients in comparison to the weakly hyperbolic case. Therefore we shall suppose $k=0$ in (3) and assume the following instead of (2).

$$
a_{i}(t) \in C^{\alpha}([0, T]) \quad \text { with } \quad 0 \leq \alpha \leq 1 \quad(i=1, \cdots, n) .
$$

Then we can prove the following theorem.

Theorem 2. Let $T>0, \mu_{0}>0$. The coefficients satisfy $(2)^{\prime}$, (3) with $k=0$ and (9). Then for any $u_{0}, u_{1}$ and $u_{2} \in \gamma^{3}$, the Cauchy problem (1) has a unique (global) solution $u \in C^{3}\left([0, T], \gamma^{s}\right)$, provided

$$
1 \leq s<\frac{1}{1-\alpha}
$$

Moreover when $u_{0}, u_{1}$ and $u_{2} \in \gamma_{0}^{s}(s>1)$, there exist the constant $\nu>0$ and the positive function $\mu(t)$ satisfying $\mu(0)=\mu_{0}$, such that for ${ }^{\forall} \xi \in \mathbb{R}_{\xi}^{n}$

$$
e^{\mu(t)\langle\xi\rangle_{\nu}^{\frac{1}{3}}}\left(\langle\xi\rangle_{\nu}^{2}|\widehat{u}|+\langle\xi\rangle_{\nu}\left|\widehat{u}_{t}\right|+\left|\widehat{u}_{t t}\right|\right) \leq{ }^{\exists} C e^{\mu_{0}\left\langle\xi^{\frac{1}{s}}\right.}\left(\langle\xi\rangle_{\nu}^{2}\left|\widehat{u}_{0}\right|+\langle\xi\rangle_{\nu}\left|\widehat{u}_{1}\right|+\left|\widehat{u}_{2}\right|\right) .
$$


As it is well known, the order (dimension) of the strictly hyperbolic equations (systems) is independent of the range of the Gevrey exponent $s$. Therefore the range (10) coincides the results of [2] for the strictly hyperbolic equations of second order and [1] for the strictly hyperbolic equations of the higher order and [8] for the strictly hyperbolic systems. In the strictly hyperbolic case we can not improve the assumption of the regularity for the coefficients $a_{i}(t)$ (see (2) with $k=0$ and $\left.(2)^{\prime}\right)$. Thus Theorem 2 is included by the results of $[1],[8]$.

We also consider the more general equation of third order in $[0, T] \times \mathbb{R}_{x}^{n}$

$$
\left\{\begin{array}{l}
L u=\sum_{i, j=1}^{n} c_{\imath \jmath}(t) u_{x_{t} x}+\sum_{i=1}^{n} d_{i}(t) u_{t x_{t}}+e(t) u_{t t}+\sum_{i=1}^{n} f_{i}(t) u_{x_{t}}+g(t) u_{t}+h(t) u \\
u(0, x)=u_{0}(x), \quad u_{t}(0, x)=u_{1}(x), \quad u_{t t}(0, x)=u_{2}(x),
\end{array}\right.
$$

where $L$ is the operator defined as $L=\partial_{t}^{3}+\sum_{t=1}^{n} a_{1}(t) \partial_{t}^{2} \partial_{x_{1}}+\sum_{t=1}^{n} b_{t j}(t) \partial_{t} \partial_{x i x,}^{2}$, and the coefficients of the lower terms satisfy

$$
c_{i j}(t), d_{i}(t), e(t), f_{l}(t), g(t), h(t) \in L^{1}([0, T])(i, j=1, \cdots, n) .
$$

In this case we also assume the restricted type of the weakly hyperbolic condition (4). Generally the lower terms influence on the wellposedness of the Cauthy problem for the weakly hyperbolic equations (see [3], [5], [9], [13], etc.). Therefore we shall assume the following conditions corresponding to a sort of Oleinik condition for the weakly hyperbolic equation of third order (12).

$$
\begin{gathered}
\left|\sum_{i, j=1}^{n} c_{i j}(t) \xi_{i} \xi_{j}\right| \leq \sigma_{1}(t, \xi)\left\{-\sum_{i, j=1}^{n} b_{i j}(t) \xi_{i} \xi_{j}\right\}^{\beta_{1}}, \\
\left|\sum_{i=1}^{n} d_{i}(t) \xi_{\imath}\right| \leq \sigma_{2}(t, \xi)\left\{\left(\sum_{i=1}^{n} a_{i}(t) \xi_{\imath}\right)^{2}-4 \sum_{i, j=1}^{n} b_{i j}(t) \xi_{l} \xi_{j}\right\}^{\beta_{2}}, \\
\left|\sum_{i, j=1}^{n} f_{i}(t) \xi_{\imath}\right| \leq \sigma_{3}(t, \xi)\left\{-\sum_{i, j=1}^{n} b_{\imath j}(t) \xi_{i} \xi_{j}\right\}^{\beta_{3}},
\end{gathered}
$$

for some $\sigma_{1}(t, \xi), \sigma_{2}(t, \xi)$ and $\sigma_{3}(t, \xi)$ such that

$$
\begin{gathered}
\sup _{\xi \in \mathbb{R}_{\xi}^{n}} \int_{0}^{T} \sigma_{1}(t, \xi)\langle\xi\rangle^{2 \beta_{1}-2} d t<+\infty . \\
\sup _{\xi \in \mathbb{R}_{\xi}^{n}} \int_{0}^{T} \sigma_{l}(t, \xi)\langle\xi\rangle^{2 \beta_{l}-1} d t<+\infty(l=2,3) .
\end{gathered}
$$

Then we can prove the following corollary. 
Corollary 3. Let $T>0, \mu_{0}>0$, and $\beta_{1} \in[0,1], \beta_{2} \in\left[0, \frac{1}{2}\right]$ and $\beta_{3} \in\left[0, \frac{1}{2}\right]$. The coefficients of the principal part satisfy (2). (3) and (4). Furthermore the coefficients of the lower terms satisfy (13), (14), (15) and (16). Then for any $u_{0}, u_{1}$ and $u_{2} \in \gamma^{s}$, the Cauchy problem (12) has a unique (global) solution $u \in$ $C^{3}\left([0, T], \gamma^{s}\right)$, provided

$$
1 \leq s<1+\min \left\{\frac{k+\alpha}{2}, \frac{1}{2-2 \beta_{1}}, \frac{1}{1-2 \beta_{2}}, \frac{2}{1-2 \beta_{3}}\right\}
$$

Moreover when $u_{0}, u_{1}$ and $u_{2} \in \gamma_{0}^{s}(s>1)$, there exist the constant $\nu>0$ and the positive function $\mu(t)$ satisfying $\mu(0)=\mu_{0}$, such that for ${ }^{\forall} \xi \in \mathbb{R}_{\xi}^{n}$

$$
e^{\mu(t)\langle\xi\rangle_{\nu}^{\frac{1}{\nu}}}\left(\langle\xi\rangle_{\nu}^{2 \omega}\left|\widehat{u_{1}}+\langle\xi\rangle\right\rangle_{\nu}^{\omega}\left|\widehat{u}_{t}\right|+\left|\widehat{u}_{t t}\right|\right) \leq{ }^{\exists} C e^{\mu_{0}\left\langle\zeta^{\xi}\right\rangle_{\nu}^{\frac{1}{\nu}}}\left(\langle\xi\rangle_{\nu}^{2}\left|\widehat{u}_{0}\right|+\langle\xi\rangle_{\nu}\left|\widehat{u}_{1}\right|+\left|\widehat{u}_{2}\right|\right),
$$

where

$$
\omega=\max \left\{\frac{2}{2+k+\alpha}, \frac{2-2 \beta_{1}}{3-2 \beta_{1}}, \frac{1-2 \beta_{2}}{2-2 \beta_{2}}, \frac{1-2 \beta_{3}}{3-2 \beta_{3}}\right\}
$$

Remark 4. From this corollary we can see the followings.

i) for $\beta_{1}=1, \beta_{2}=\frac{1}{2}$ and $\beta_{3}=\frac{1}{2}$ we can get the same result as Theorem 1 .

ii) the lower term $\sum_{\imath, j=1}^{n} c_{\imath \jmath}(t)_{u_{x} x}$, influences on the $\gamma^{s}$-wellposedness if $s \geq \frac{3}{2}$.

iii) the lower term $\sum_{t=1}^{n} d_{1}(t) u_{t x_{t}}$ influences on the $\gamma^{s}$-wellposedness if $s \geq 2$.

iv) the lower term $\sum_{\imath=1}^{n} f_{\imath}(t) u_{x}$ influences on the $\gamma^{s}$-wellposedness if $s \geq 3$.

v) the lower terms $e(t) u_{t}, g(t) u_{t}$ and $h(t) u$ don't influence on the $\gamma^{s}$-wellposedness.

Our proofs of the theorems and the corollary are based on the methods of the energies for the hyperbolic equations of third order (see the definitions of the energies (50) for the weakly hyperbolic case and (57) for the strictly hyperbolic case). Thanks to these energies, we can get the inequalities (7), (11) and (20) which denote the differences between the regularity of the solution and the regularity of the initial data.

\section{Acknowledgments}

The author wishes to thank Prof. K. Kajitani and Prof. S. Wakabayashi for many useful advices. Moreover the author also wishes to thank the referee for the invaluable remarks. 


\section{Notations}

$$
\begin{aligned}
\langle\xi\rangle_{\nu} & =\left(|\xi|^{2}+\nu^{2}\right)^{\frac{1}{2}} \quad(\nu>0) . \quad\langle\xi\rangle=\left(|\xi|^{2}+1\right)^{\frac{1}{2}}\left(=\langle\xi\rangle_{1}\right) . \\
\hat{f}(\xi) & =\mathscr{F}[f](\xi)=\int e^{-i x \cdot \xi} f(x) d x .
\end{aligned}
$$

$C^{k+\alpha}([0, T])\left(k \in \mathbb{N}^{1}, 0 \leq \alpha \leq 1\right)$ is the space of functions $f(t)$ having $k$ derivatives continuous, and the $k$-th derivative Hölder continuous with exponent $\alpha$ on $[0, T]$.

$r^{s}\left(\mathbb{R}_{x}^{n}\right)(s \geq 1)$ is the space of Gevrey functions $f(x)$ satisfying for any compact set $K \subset \mathbb{R}^{n}$,

$$
\sup _{x \in K}\left|D^{\alpha} f(x)\right| \leq C_{K} \rho_{K}^{|\alpha|}|\alpha|^{s} ! \quad \text { for }{ }^{\forall} \alpha \in \mathbb{N}^{n} .
$$

$\gamma_{0}^{s}\left(\mathbb{R}_{x}^{n}\right)(s>1)$ is the space of Gevrey functions $f(x)$ or the order $s$ having compact support.

\section{§ 2. Proof of Theorem 1}

When $s=1$, the problem (1) is well-posed in $\gamma^{1}$ which is the topological vector space of analytic functions on $\mathbb{R}^{n}$ (see [2] for the weakly hyperbolic equations of second order and see [7] for the weakly hyperbolic systems including the third order equations). Therefore we can suppose $s>1$ for the proof.

In virtue of Holmgren's theorem we get the uniqueness of solutions to (1) and can suppose that $u_{0}(x), u_{1}(x)$ and $u_{2}(x)$ belong to $\gamma_{0}^{s}$. Hence by PaleyWiener theorem we shall assume that

$$
\sup _{\xi \in \mathbb{R}_{\xi}^{n}} e^{\mu_{0}\langle\xi\rangle_{\nu}^{\frac{1}{\delta}}}\left(\langle\xi\rangle_{\nu}^{2}\left|\widehat{u}_{0}\right|+\langle\xi\rangle_{\nu}\left|\widehat{u}_{1}\right|+\left|\widehat{u_{2}}\right|\right)<+\infty .
$$

Moreover Ovciannikov theorem gives the existence of solutions (see [3], [8], [12]). Our task is to investigate the regularity for $x$ of the solution, namely, to derive the energy inequality (7).

By Fourier transform the Cauchy problem (1) is changed to

$$
\left\{\begin{array}{l}
v_{t t t}+i a(t, \xi) v_{t t}-b(t, \xi) v_{t}=0 \\
v(0, \xi)=v_{0}(\xi), \quad v_{t}(0, \xi)=v_{1}(\xi), \quad v_{t t}(0, \xi)=v_{2}(\xi)
\end{array}\right.
$$

where $v=\widehat{u}$, and $v_{l}=\widehat{u}_{l}(l=0,1,2)$, and $a(t, \xi)=\sum_{l=1}^{n} a_{l}(t) \xi_{i}, b(t, \xi)=\sum_{l, j=1}^{n}$ $b_{\imath}(t) \xi_{1} \xi_{j}$.

Now we must separate the proof of Theorem 1 into three parts according to the smoothness of the coefficients. 


\subsection{Case of $k=0$}

We first treat the case of $k=0$ which implies that both coefficients $a(t, \xi)$ and $b(t, \xi)$ belong to Hölder classes in $t$. Since these coefficients are not differentiable, they can not enter into the definition of the energy directly. Therefore we shall regularize them as follows.

$$
\begin{aligned}
& a_{\varepsilon}(t, \xi)=\frac{1}{\varepsilon} \int_{-\infty}^{\infty} a(t+\tau, \xi) \varphi\left(\frac{\tau}{\varepsilon}\right) d \tau, \\
& b_{\varepsilon}(t, \xi)=\frac{1}{\varepsilon} \int_{-\infty}^{\infty} b(t+\tau, \xi) \varphi\left(\frac{\tau}{\varepsilon}\right) d \tau,
\end{aligned}
$$

$(0<\varepsilon<1)$, where $\varphi(t) \in C_{0}^{\infty}\left(\mathbb{R}_{t}^{1}\right)$ satisfies $0 \leq \varphi(t)<\infty$ and $\int_{-\infty}^{\infty} \varphi(t) d t=1$.

Then there exists $C_{0}>0$ such that for ${ }^{\forall} \xi \in \mathbb{R}_{n}^{\xi}$

$$
\begin{array}{ll}
\left|a_{\varepsilon}^{\prime}(t, \xi)\right| \leq C_{0} \varepsilon^{\frac{\alpha}{2}-1}\langle\xi\rangle_{\nu,} & \left|a_{\varepsilon}(t, \xi)-a(t, \xi)\right| \leq C_{0} \varepsilon^{\frac{\alpha}{2}}\langle\xi\rangle_{\nu}, \\
\left|b_{\varepsilon}^{\prime}(t, \xi)\right| \leq C_{0} \varepsilon^{\alpha-1}\langle\xi\rangle_{\nu}^{2}, & \left|b_{\varepsilon}(t, \xi)-b(t, \xi)\right| \leq C_{0} \varepsilon^{\alpha}\langle\xi\rangle_{\nu}^{2} .
\end{array}
$$

With the coefficients $a_{\varepsilon}(t, \xi), b_{\varepsilon}(t, \xi)$ we shall define the following energy.

$$
\begin{aligned}
& E_{\varepsilon, \nu}(t, \xi)^{2}=e^{\rho(t)\langle(\xi) x}\left\{\left.\left|v_{t t}+i a_{\varepsilon}(t, \xi) v_{t}-b_{\varepsilon}(t, \xi) v+\varepsilon^{\alpha}\langle\xi\rangle\right\rangle_{\nu} v\right|^{2}\right. \\
& \left.\left.+\left|v_{t t}+\frac{i}{2} a_{\varepsilon}(t, \xi) v_{t}\right|^{2}+\left(\frac{a_{\varepsilon}(t, \xi)^{2}}{4}-b_{\varepsilon}(t, \xi)+\varepsilon^{\alpha}\langle\xi\rangle\right\rangle_{\nu}\right)\left|v_{t}\right|^{2}\right\} .
\end{aligned}
$$

Here $\rho(t)$ is positive and determined later on. Thanks to the conditions (4) and the term $\varepsilon^{\alpha}\langle\xi\rangle_{\nu}^{2}$, this energy can be bounded from below by the absolute values of $v, v_{t}$ and $v_{t t}$. While we can also easily see that this energy is bounded (from above) by the absolute values of them. Therefore the energy inequality based on

(28) can be changed into the one based on the absolute values of them.

Differentiating (28) in $t$, by (23) we get

$$
\begin{aligned}
& \frac{d}{d t}\left(E_{\varepsilon, \nu}^{2}\right)=\rho^{\prime}(t)\langle\xi\rangle{ }_{\nu}^{\varkappa} E_{\varepsilon, \nu}^{2} \\
& +2 e^{\rho(t), \xi i \nu} \mathfrak{R}\left(i\left(a_{\varepsilon}-a\right) v_{t t}-\left(b_{\varepsilon}-b\right) v_{t}+\varepsilon^{\alpha}\langle\xi\rangle_{\nu}^{2} v_{t}+i a_{\varepsilon}^{\prime} v_{t}-b_{\varepsilon}^{\prime} v .\right. \\
& \left.v_{t t}+i a_{\varepsilon} v_{t}-b_{\varepsilon} v+\varepsilon^{\alpha}\langle\xi\rangle_{\nu}^{2} v\right) \\
& +2 e^{\rho(t)\left\langle\xi^{\prime}{ }_{\nu} x\right.} \Re\left(-i a v_{t t}+b v_{t}+\frac{i}{2} a_{\varepsilon} v_{t t}+\frac{i}{2} a_{\varepsilon}^{\prime} v_{t}, v_{t t}+\frac{i}{2} a_{\varepsilon} v_{t}\right) \\
& +2\left(\frac{a_{\varepsilon}^{2}}{4}-b_{\varepsilon}+\varepsilon^{\alpha}\langle\xi\rangle_{\nu}^{2}\right) e^{\rho(t)\langle\xi\rangle\rangle_{\nu}} \Re\left(v_{t}, v_{t t}\right)+e^{\rho(1)\langle\xi\rangle x}\left(\frac{1}{2} a_{\varepsilon} a_{\varepsilon}^{\prime}-b_{\varepsilon}^{\prime}\right)\left|v_{t}^{\prime}\right|^{2} \\
& \left(\equiv \rho^{\prime}(t)\langle\xi\rangle_{\nu}{ }_{\nu} E_{\varepsilon, \nu}^{2}+I+I I+I I I+I V\right) \text {. }
\end{aligned}
$$

In order to further estimate the derivative of the energy, we shall pick up 
the term $I$. We first rewrite $I$ as follows.

$$
\begin{aligned}
& \text { (30) } \left.I=2\left(a_{\varepsilon}-a\right) e^{\rho(t)\left\langle\xi_{\nu} x\right.} \Re\left(i\left(v_{t t}+\frac{i}{2} a_{\varepsilon} v_{t}\right), v_{t t}+i a_{\varepsilon} v_{t}-b_{\varepsilon} v+\varepsilon^{\alpha}\langle\xi\rangle\right\rangle_{\nu}^{2} v\right)
\end{aligned}
$$

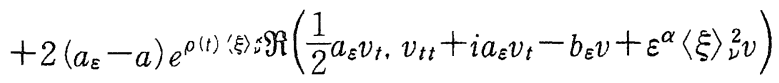

$$
\begin{aligned}
& -2\left(b_{\varepsilon}-b\right) e^{\rho(t)\left\langle\xi^{\prime}{ }^{\prime}\right.} \Re\left(\varepsilon^{\frac{\alpha}{4}}\langle\xi\rangle_{\nu}^{\frac{1}{2}} v_{t}, \varepsilon^{-\frac{\alpha}{4}}\langle\xi\rangle_{\nu}^{-\frac{1}{2}}\left(v_{t t}+i a_{s} v_{t}-b_{\varepsilon} v+\varepsilon^{\alpha}\langle\xi\rangle_{\nu}^{2} v\right)\right) \\
& +2 \varepsilon^{\alpha}\langle\xi\rangle_{\nu}^{2} e^{\rho(t)\langle\xi\rangle} \stackrel{\Re}{\wedge}\left(\varepsilon^{\frac{\alpha}{4}}\langle\xi\rangle_{\nu}^{\frac{1}{2}} v_{t}, \varepsilon^{-\frac{\alpha}{4}}\langle\xi\rangle_{\nu}^{-\frac{1}{2}}\left(v_{t t}+i a_{\varepsilon} v_{t}-b_{\varepsilon} v+\varepsilon^{\alpha}\langle\xi\rangle_{\nu}^{2} v\right)\right) \\
& +2 a_{\varepsilon}^{\prime} e^{\left.\rho(t), \varepsilon_{\nu}\right\rangle_{\Re}}\left(i \varepsilon^{\frac{\alpha}{4}}\langle\xi\rangle_{\nu}^{\frac{1}{2}} v_{t}, \varepsilon^{-\frac{\alpha}{4}}\langle\xi\rangle_{\nu}^{-\frac{1}{2}}\left(v_{t t}+i a_{\varepsilon} v_{t}-b_{\varepsilon} v+\varepsilon^{\alpha}\langle\xi\rangle_{\nu}^{2} v\right)\right) \\
& +2 \frac{-b_{\varepsilon}^{\prime}}{-b_{\varepsilon}+\varepsilon^{\alpha}\langle\xi\rangle_{\nu}^{2}} e^{\rho(t)\langle\xi\rangle} \stackrel{R}{R}\left(-v_{t t}-\frac{i}{2} a_{\varepsilon} v_{t}, v_{t t}+i a_{\varepsilon} v_{t}-b_{\varepsilon} v+\varepsilon^{\alpha}\langle\xi\rangle_{\nu}^{2} v\right) \\
& +2 \frac{-b_{\varepsilon}^{\prime}}{-b_{\varepsilon}+\varepsilon^{\alpha}\langle\xi\rangle_{\nu}^{2}} e^{\rho\left(r^{\prime}\langle\xi)^{*}\right.} \Re\left(-\frac{i}{2} a_{\varepsilon} v_{t}, v_{t t}+i a_{\varepsilon} v_{t}-b_{\varepsilon} v^{\prime}+\varepsilon^{\alpha}\langle\xi\rangle_{\nu} \nu^{\prime}\right) \\
& +\left.2 \frac{-b_{\varepsilon}^{\prime}}{-b_{\varepsilon}+\varepsilon^{\alpha}\langle\xi\rangle_{\nu}} e^{\rho\left(t^{\prime}\langle\xi)^{\prime}{ }^{\prime}\right.}\right|_{v^{\prime} t t}+i a_{\varepsilon} v_{t}-b_{\varepsilon} v+\left.\varepsilon^{\alpha}\langle\xi\rangle_{\nu}^{2} v\right|^{2} \text {. }
\end{aligned}
$$

For this expression, we remark that the denominator or the fraction in the last three terms is not zero. since $-b_{\varepsilon}$ is non-negative by the condition (4).

Noting the definition of the energy (28), by (26). (27), (30) we obtain

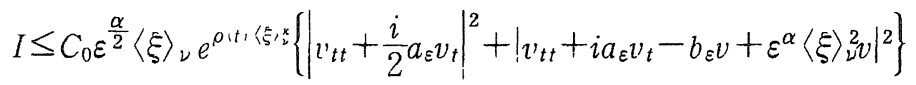

$$
\begin{aligned}
& +C_{0} \varepsilon^{\frac{\alpha}{2}}\langle\xi\rangle_{\nu} e^{\rho(t)\langle\xi\rangle x}\left\{\frac{1}{4} a_{\varepsilon}^{2}\left|v_{t}\right|^{2}+\left|v_{t t}+i a_{\varepsilon} v_{t}-b_{\varepsilon} v^{\prime}+\varepsilon^{\alpha}\langle\xi\rangle_{\nu}{ }^{2}\right|^{2}\right\} \\
& +C_{0} \varepsilon^{\alpha}\langle\xi\rangle_{\nu}{ }_{\nu} e^{\rho(t)\left\langle\xi^{\prime \prime x}\right.}\left\{\varepsilon^{\frac{\alpha}{2}}\langle\xi\rangle_{\nu}\left|v_{t}\right|^{2}+\varepsilon^{-\frac{\alpha}{2}}\langle\xi\rangle_{\nu}{ }^{-1}\left|v_{t t}+i a_{\varepsilon} v_{t}-b_{\varepsilon} v+\varepsilon^{\alpha}\langle\xi\rangle_{\nu}{ }_{\nu}\right|^{2}\right\} \\
& +\varepsilon^{\alpha}\langle\xi\rangle_{\nu}^{2} e^{\rho(t)\langle\xi}{ }^{\times}\left\{\varepsilon^{\frac{\alpha}{2}}\langle\xi\rangle_{\nu}\left|v_{t}\right|^{2}+\varepsilon^{-\frac{\alpha}{2}}\langle\xi\rangle_{\nu}{ }^{-1}\left|v_{t t}+i a_{\varepsilon} v_{t}-b_{\varepsilon} \nu+\varepsilon^{\alpha}\langle\xi\rangle_{\nu} v\right|{ }^{2}\right\} \\
& +C_{0} \varepsilon^{\frac{\alpha}{2}-1}\langle\xi\rangle_{\nu} e^{\rho^{(t)}\langle(\xi) x}\left\{\varepsilon^{\frac{\alpha}{2}}\langle\xi\rangle_{\nu}\left|v_{t}\right|^{2}+\varepsilon^{-\frac{\alpha}{2}}\langle\xi\rangle_{\nu}{ }^{-1}\left|v_{t t}+i a_{\varepsilon} v_{t}-b_{\varepsilon} v+\varepsilon^{\alpha}\langle\xi\rangle_{\nu} v\right|^{2}\right\} \\
& +C_{0} \varepsilon^{-1} e^{\rho(t)\langle\hat{\langle}\rangle \nu}\left\{\left|v_{t t}+\frac{i}{2} a_{\varepsilon} v_{t}\right|^{2}+\left.\left|v_{t t}+i a_{\varepsilon} v_{t}-b_{\varepsilon} v+\varepsilon^{\alpha}\langle\xi\rangle\right\rangle_{\nu}^{2}\right|^{2}\right\} \\
& +C_{0} \varepsilon^{-1} e^{\rho(t) \xi) *}\left\{\frac{1}{4} a_{\varepsilon}^{2}\left|v_{t}\right|^{2}+\left|v_{t t}+i a_{\varepsilon} v_{t}-b_{\varepsilon} v+\varepsilon^{\alpha}\langle\xi\rangle{ }_{\nu}^{2}\right|^{2}\right\} \\
& +\left.2 C_{0} \varepsilon^{-1} e^{\rho(t)\langle(\xi) \nu}\right|_{v^{\prime} t t}+i a_{\varepsilon} v_{t}-b_{\varepsilon} v+\left.\varepsilon^{\alpha}\langle\xi\rangle_{\nu l}^{2}\right|^{2} \\
& \leq C_{0} \varepsilon^{\frac{\alpha}{2}}\langle\xi\rangle_{\nu} E_{\varepsilon, \nu}^{2}+C_{0} \varepsilon^{\frac{\alpha}{2}}\langle\xi\rangle_{\nu} E_{\varepsilon, \nu}^{2}+C_{0} \varepsilon^{\frac{\alpha}{2}}\langle\xi\rangle_{\nu} E_{\varepsilon, \nu}^{2}+\varepsilon^{\frac{\alpha}{2}}\langle\xi\rangle_{\nu} E_{\varepsilon, \nu}^{2} \\
& +C_{0} \varepsilon^{-1} E_{\varepsilon, \nu}^{2}+C_{0} \varepsilon^{-1} E_{\varepsilon, \nu}^{2}+C_{0} \varepsilon^{-1} E_{\varepsilon, \nu}^{2}+2 C_{0} \varepsilon^{-1} E_{\varepsilon, \nu}^{2} \\
& =\left(3 C_{0}+1\right) \varepsilon^{\frac{\alpha}{2}}\langle\xi\rangle_{\nu} E_{\varepsilon, \nu}^{2}+5 C_{0} \varepsilon^{-1} E_{\varepsilon, \nu}^{2} \text {, }
\end{aligned}
$$

here we used $2 \Re\left(z_{1}, z_{2}\right) \leq\left|z_{1}\right|^{2}+\left|z_{2}\right|^{2}$ for $\stackrel{\forall}{z_{1}}, \stackrel{\forall}{z_{2}} \in \mathbb{C}^{1}$.

Secondly we shall estimate the other terms. From the definition of the energy (28) we also find that 


$$
E_{\varepsilon, \nu}^{2} \geq e^{\rho(t)\langle\xi\rangle \nu}\left\{\frac{1}{4} a_{\varepsilon}^{2}+\varepsilon^{\alpha}\langle\xi\rangle_{\nu}^{2}\right\}\left|v_{t}\right|^{2} \geq e^{\rho(t)\langle\xi\rangle}\left|a_{\varepsilon}\right| \varepsilon^{\frac{\alpha}{2}}\langle\xi\rangle \nu_{\nu}\left|v_{t}\right|^{2}
$$

Dealing with the terms $I I, I I I$ and $I V$ together, by (26), (27), (32) we obtain

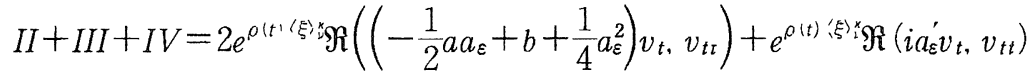

$$
\begin{aligned}
& +\frac{1}{2} e^{\rho(t)\left\langle\xi^{\prime}\right.} a_{\varepsilon}^{\prime} a_{\varepsilon}\left|v_{t}\right|^{2}+I I I+I V
\end{aligned}
$$

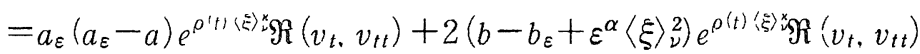

$$
\begin{aligned}
& +a_{\varepsilon} a_{\varepsilon}^{\prime} e^{\rho(t)\langle\xi}\left|v_{t}\right|^{2}+\left(-b_{\varepsilon}^{\prime}\right) e^{\rho(t)\left\langle\xi \xi^{\prime} x\right.}\left|v_{t}\right|^{2}+a_{\varepsilon}^{\prime} e^{\rho(t)\rangle \xi\rangle} \Re\left(i v_{t}, v_{t t}\right) \\
& =2\left(a_{\varepsilon}-a\right) e^{\left.\rho^{\prime} t\right)(\xi)^{\prime} x_{S}} \mathfrak{R}\left(\frac{1}{2} a_{\varepsilon} v^{\prime} t, v_{t t}+\frac{i}{2} a_{\varepsilon} v_{t}\right) \\
& +2\left(b-b_{\varepsilon}+\varepsilon^{\alpha}\langle\xi\rangle_{\nu}^{2}\right) e^{\rho(t)\langle\xi\rangle \times} \Re\left(\varepsilon^{\frac{\alpha}{4}}\langle\xi\rangle_{\nu}^{\frac{1}{2}} v_{t}^{\prime}, \varepsilon^{-\frac{\alpha}{4}}\langle\xi\rangle_{\nu}^{-\frac{1}{2}}\left(v_{t t}+\frac{i}{2} a_{\varepsilon} v_{t}\right)\right) \\
& +a_{\varepsilon}^{\prime} \varepsilon^{-\frac{\alpha}{2}}\langle\xi\rangle_{\nu}{ }_{\nu}^{-1} e^{\rho(t)\left\langle\xi^{\prime}\right)^{x}} a_{\varepsilon} \varepsilon^{\frac{\alpha}{2}}\langle\xi\rangle_{\nu}\left|v_{t}\right|^{2}+\left(-b_{\varepsilon}\right) \varepsilon^{-\alpha}\langle\xi\rangle_{\nu}{ }_{\nu}^{-2} e^{\left.\rho(t) \xi^{\xi}\right\rangle^{x}} \varepsilon^{\alpha}\langle\xi\rangle_{\nu}{ }_{\nu}\left|v_{t}\right|^{2} \\
& +2 a_{\varepsilon}^{\prime} \varepsilon^{\rho(t)\left\langle\xi^{\prime}\right)} \Re\left(\frac{i}{2} \varepsilon^{\frac{\alpha}{4}}\langle\xi\rangle_{\nu}^{\frac{1}{2}} v_{t}, \varepsilon^{-\frac{\alpha}{4}}\langle\xi\rangle_{\nu}^{-\frac{1}{2}}\left(v_{t t}+\frac{i}{2} a_{\varepsilon} v_{t}\right)\right) \\
& +2 a_{\varepsilon}^{\prime} c^{\rho(t)\langle\bar{s} x} \Re\left(\frac{i}{2} v_{t,}-\frac{i}{2} a_{\varepsilon} v_{t}\right)\left(=-\frac{1}{2} \times “ \text { third term” }\right) \\
& \leq C_{0} \varepsilon^{\frac{\alpha}{2}}\langle\xi\rangle_{\nu} e^{\rho(t)\langle\xi}:\left\{\frac{1}{4} a_{\varepsilon}^{2}\left|v_{t}\right|^{2}+\left|v_{t t}+\frac{i}{2} a_{\varepsilon} v_{t}\right|^{2}\right\} \\
& +\left(C_{0}+1\right) \varepsilon^{\alpha}\langle\xi\rangle_{\nu}^{2} e^{\rho(t)\langle\xi\rangle x}\left\{\varepsilon^{\frac{\alpha}{2}}\langle\xi\rangle_{\nu}\left|v_{t}\right|^{2}+\varepsilon^{-\frac{\alpha}{2}}\langle\xi\rangle_{\nu}^{-1}\left|v_{t t}+\frac{i}{2} a_{\varepsilon} v_{t}\right|^{2}\right\} \\
& +\frac{C_{0}}{2} \varepsilon^{\frac{\alpha}{2}-1}\langle\xi\rangle_{\nu} \varepsilon^{-\frac{\alpha}{2}}\langle\xi\rangle_{\nu}^{-1} e^{\rho(t) \xi)^{*}}\left|a_{\varepsilon}\right| \varepsilon^{\frac{\alpha}{2}}\langle\xi\rangle_{\nu}\left|v_{t}\right|^{2} \\
& +C_{0} \varepsilon^{\alpha-1}\langle\xi\rangle_{\nu}^{2} \varepsilon^{-\alpha}\langle\xi\rangle_{\nu}^{-2} e^{\rho(t)\left\langle\xi^{\prime \prime}{ }_{\nu}^{\alpha}\right.} \varepsilon^{\alpha}\langle\xi\rangle{ }_{\nu}\left|v_{t}\right|^{2} \\
& +C_{0} \varepsilon^{\frac{\alpha}{2}-1}\langle\xi\rangle_{\nu} e^{\left.\rho^{\prime} t\right)^{\prime} \xi^{\prime \prime}}\left\{\varepsilon^{\frac{\alpha}{2}}\langle\xi\rangle_{\nu}\left|v_{t}\right|^{2}+\varepsilon^{-\frac{\alpha}{2}}\langle\xi\rangle_{\nu}^{-1}\left|v_{t t}+\frac{i}{2} a_{\varepsilon} v_{t}\right|^{2}\right\} \\
& \leq C_{0} \varepsilon^{\frac{\alpha}{2}}\langle\xi\rangle_{\nu} E_{\varepsilon, \nu}^{2}+\left(C_{0}+1\right) \varepsilon^{\frac{\alpha}{2}}\langle\xi\rangle_{\nu} E_{\varepsilon, \nu}^{2}+\frac{C_{0}}{2} \varepsilon^{-1} E_{\varepsilon, \nu}^{2}+C_{0} \varepsilon^{-1} E_{\varepsilon, \nu}^{2}+C_{0} \varepsilon^{-1} E_{\varepsilon, \nu}^{2} \\
& =\left(2 C_{0}+1\right) \varepsilon^{\frac{\alpha}{2}}\langle\xi\rangle_{\nu} E_{\varepsilon, \nu}^{2}+\frac{5}{2} C_{0} \varepsilon^{-1} E_{\varepsilon, \nu}^{2},
\end{aligned}
$$

here we used $\Re(i z, z)=\Re\left\{i|z|^{2}\right\}=0$ for ${ }^{\forall} \in \mathbb{C}^{1}$.

Therefore by (29), (31), (33) we have the estimate

$$
\frac{d}{d t}\left(E_{\varepsilon, \nu}^{2}\right) \leq \rho^{\prime}(t)\langle\xi\rangle_{\nu}^{\wedge} E_{\varepsilon, \nu}^{2}+C_{1} \varepsilon^{\frac{\alpha}{2}}\langle\xi\rangle_{\nu} E_{\varepsilon, \nu}^{2}+C_{2} \varepsilon^{-1} E_{\varepsilon, \nu}^{2},
$$

where $C_{1}=5 C_{0}+2, C_{2}=\frac{15}{2} C_{0}$.

Thus Gronwall's inequality yields 


$$
E_{\varepsilon, \nu}^{2}(t, \xi) \leq E_{\varepsilon, \nu}^{2}(0, \xi) \exp \left[\int_{0}^{t}\left\{\rho^{\prime}(\tau)\langle\xi\rangle_{\nu}{ }^{x}+C_{1} \varepsilon^{\frac{\alpha}{2}}\langle\xi\rangle_{\nu}+C_{2} \varepsilon^{-1}\right\}_{d \tau}\right] \text { for }{ }^{\forall} t \in[0, T]
$$

Since $s\left(=\kappa^{-1}\right)<1+\frac{\alpha}{2}$, we find $\frac{2}{2+\alpha}-\kappa<0$ and $\langle\xi\rangle_{\nu}^{\frac{2}{2+\alpha}-\kappa} \leq \nu^{\frac{2}{2+\alpha}-\kappa}$.

Hence we take $\varepsilon=\langle\xi\rangle_{\nu}-\frac{2}{2+\alpha}$ and obtain

$$
\begin{aligned}
E_{\varepsilon, \nu}^{2}(t, \xi) & \leq E_{\varepsilon, \nu}^{2}(0, \xi) \exp \left[\langle\xi\rangle_{\nu}^{x} \int_{0}^{t}\left\{\rho^{\prime}(\tau)+\left(C_{1}+C_{2}\right) \nu^{\frac{2}{2+\alpha}-x}\right\} d \tau\right] \\
& =E_{\varepsilon, \nu}^{2}(0, \xi) \exp \left[\langle\xi\rangle_{\nu}^{x}\left\{\rho(t)-\rho_{0}+C_{3} t \nu^{\frac{2}{2+\alpha}-x}\right\}\right] \text { for }{ }^{{ }^{\prime}} t \in[0, T],
\end{aligned}
$$

where $\rho_{0}=\rho(0), C_{3}=C_{1}+C_{2}$.

Moreover we determine $\rho(t)=\rho_{0}-C_{3} t \nu^{\frac{2}{2+\alpha}-x}$, and choose $\nu>0$ such that $\rho(T)\left(=\rho_{0}-C_{3} T \nu^{\frac{2}{2+\alpha}-x}\right)>0$ for any given $T>0$.

Finally we have the energy inequality

$$
E_{\varepsilon, \nu}^{2}(t, \xi) \leq E_{\varepsilon, \nu}^{2}(0 . \xi) \quad \text { for }{ }^{\forall} t \in[0, T] \text { and }{ }^{\forall} \xi \in \mathbb{R}_{\xi}^{n} .
$$

\subsection{Case of $k=\mathbb{1}$}

We next treat the case of $k=1$ which implies that the coefficient $a(t, \xi)$ belongs to Hölder class while $b(t, \xi)$ belongs to $C^{1}$ class in $t$ at least. Therefore we shall only regularize the coefficient $a(t, \xi)$ as (24), and get

$$
\left|a_{\varepsilon}^{\prime}(t, \xi)\right| \leq C_{0} \varepsilon^{\frac{\alpha+1}{2}-1}\langle\xi\rangle_{\nu}, \quad\left|a_{\varepsilon}(t, \xi)-a(t, \xi)\right| \leq C_{0} \varepsilon^{\frac{\alpha+1}{2}}\langle\xi\rangle_{\nu}
$$

With the coefficients $a_{\varepsilon}(t, \xi), b(t, \xi)$ we shall define the following energy.

$$
\begin{aligned}
& E_{\varepsilon, \nu}(t, \xi)^{2}=e^{\rho(t)\langle\xi, x},\left\{\left.\left|v_{t t}+i a_{\varepsilon}(t, \xi) v_{t}-b(t, \xi) v+\varepsilon^{1+\alpha}\langle\xi\rangle\right\rangle_{\nu}{ }^{2}\right|^{2}\right. \\
&\left.+\left|v_{t t}+\frac{i}{2} a_{\varepsilon}(t, \xi) v_{t}\right|^{2}+\left(\frac{a_{\varepsilon}(t, \xi)^{2}}{4}-b(t, \xi)+\varepsilon^{1+\alpha}\langle\xi\rangle_{\nu} 2\right)\left|v_{t}\right|^{2}\right\} .
\end{aligned}
$$

Differentiating (36) in $t$, by (23) we get

$$
\begin{aligned}
& \frac{d}{d t}\left(E_{\varepsilon, \nu}^{2}\right)=\rho^{\prime}(t)\langle\xi\rangle_{\nu}^{\mu} E_{\varepsilon, \nu}^{2}+2 e^{\rho(t)\langle\xi\rangle}{ }_{x} \Re\left(i\left(a_{\varepsilon}-a\right) v_{t t}+\varepsilon^{1+\alpha}\langle\xi\rangle_{\nu}^{2} v_{t}+i a_{\varepsilon}^{\prime} v_{t}-b^{\prime} v,\right. \\
& \left.v_{t t}+i a_{\varepsilon} v_{t}-b v+\varepsilon^{1+\alpha}\langle\xi\rangle{ }_{\nu}^{2} v\right) \\
& +2 e^{\rho(t)\langle\xi\rangle_{\nu} \varkappa} \Re\left(-i a v^{\prime} t t+b v_{t}+\frac{i}{2} a_{\varepsilon} v_{t t}+\frac{i}{2} a_{\varepsilon}^{\prime} v_{t}, v_{t t}+\frac{i}{2} a_{\varepsilon} v_{t}\right) \\
& +2\left(\frac{a_{\varepsilon}^{2}}{4}-b+\varepsilon^{1+\alpha}\langle\xi\rangle_{\nu}^{2}\right) e^{\rho\left(t^{\prime}, \xi\right) / x} \Re\left(v_{t}, v_{t t}\right)+e^{\rho(t)\langle\xi / x}\left(\frac{1}{2} a_{\varepsilon} a_{\varepsilon}^{\prime}-b^{\prime}\right)\left|v_{t}\right|^{2} \\
& \left(\equiv \rho^{\prime}(t)\langle\xi\rangle{ }_{\nu} E_{\varepsilon, \nu}^{2}+I^{\prime}+I I^{\prime}+I I I^{\prime}+I V^{\prime}\right) \text {. }
\end{aligned}
$$


Noting that

$$
\frac{-b^{\prime}}{-b+\varepsilon^{1+\alpha}\langle\xi\rangle_{\nu}^{2}} \leq \frac{\left|\left(-b+\varepsilon^{1+\alpha}\langle\xi\rangle_{\nu}^{2}\right)^{\prime}\right|}{\left(-b+\varepsilon^{1+\alpha}\langle\xi\rangle_{\nu}^{2}\right)^{1-\frac{1}{1+\alpha}} \varepsilon\langle\xi\rangle_{\nu}^{\frac{2}{1+\alpha}}}=(1+\alpha)\left|\left\{\left(-b+\varepsilon^{1+\alpha}\langle\xi\rangle_{\nu}^{2}\right)^{\frac{1}{1+\alpha}}\right\}^{\prime}\right| \varepsilon^{-1}\langle\xi\rangle_{\nu}^{-\frac{2}{1+\alpha}},
$$

similarly as the case of $k=0$, we can estimate $I^{\prime}$ and $I I^{\prime}+I I I^{\prime}+I V^{\prime}$ as follows.

$$
\begin{aligned}
& I^{\prime}=2\left(a_{\varepsilon}-a\right) e^{\rho(t)\langle\xi\rangle} x \Re\left(i\left(v_{t t}+\frac{i}{2} a_{\varepsilon} v_{t}\right), v_{t t}+i a_{\varepsilon} v_{t}-b v+\varepsilon^{1+\alpha}\langle\xi\rangle_{\nu}^{2} v\right) \\
& +2\left(a_{\varepsilon}-a\right) e^{\rho(t)\langle\xi\rangle}{ }^{\kappa} \Re\left(\frac{1}{2} a_{\varepsilon} v_{t}, v_{t t}+i a_{\varepsilon} v_{t}-b v+\varepsilon^{1+\alpha}\langle\xi\rangle_{\nu} v\right) \\
& +2 \varepsilon^{1+\alpha}\langle\xi\rangle_{\nu}^{2} e^{\rho(t)\langle\xi\rangle} \Re_{\mathcal{R}}\left(\varepsilon^{\frac{1+\alpha}{4}}\langle\xi\rangle_{\nu}^{\frac{1}{2}} v_{t}, \varepsilon^{-\frac{1+\alpha}{4}}\langle\xi\rangle_{\nu}^{-\frac{1}{2}}\left(v_{t t}+i a_{\varepsilon} v_{t}-b v+\varepsilon^{1+\alpha}\langle\xi\rangle_{\nu}^{2} v\right)\right) \\
& +2 a_{\varepsilon}^{\prime} e^{\rho(t)\langle\xi\rangle\rangle_{\Re}}\left(i \varepsilon^{\frac{1+\alpha}{4}}\langle\xi\rangle_{\nu}^{\frac{1}{2}} v_{t}, \varepsilon^{-\frac{1+\alpha}{4}}\langle\xi\rangle_{\nu}^{-\frac{1}{2}}\left(v_{t t}+i a_{\varepsilon} v_{t}-b v+\varepsilon^{1+\alpha}\langle\xi\rangle_{\nu} v\right)\right) \\
& +2 \frac{-b^{\prime}}{-b+\varepsilon^{1+\alpha}\langle\xi\rangle_{\nu}^{2}} e^{\rho(t)\langle\xi\rangle} \Re\left(-v_{t t}-\frac{i}{2} a_{\varepsilon} v_{t}, v_{t t}+i a_{\varepsilon} v_{t}-b v+\varepsilon^{1+\alpha}\langle\xi\rangle_{\nu}^{2} v\right) \\
& +2 \frac{-b^{\prime}}{-b+\varepsilon^{1+\alpha}\langle\xi\rangle_{\nu}^{2}} e^{\rho(t)\langle\xi\rangle_{n}^{\prime}} \Re\left(-\frac{i}{2} a_{\varepsilon} v_{t}, v_{t t}+i a_{\varepsilon} v_{t}-b v+\varepsilon^{1+\alpha}\langle\xi\rangle_{\nu}^{2} v\right) \\
& +2 \frac{-b^{\prime}}{-b+\varepsilon^{1+\alpha}\langle\xi\rangle_{\nu}^{2}} e^{\rho(t)\langle\xi\rangle^{\prime}}\left|v_{i t}+i a_{\varepsilon} v_{t}-b v+\varepsilon^{1+\alpha}\langle\xi\rangle_{\nu}^{2} v\right|^{2} \\
& \leq C_{0} \varepsilon^{\frac{1+\alpha}{2}}\langle\xi\rangle_{\nu} E_{\varepsilon, \nu}^{2}+C_{0} \varepsilon^{\frac{1+\alpha}{2}}\langle\xi\rangle_{\nu} E_{\varepsilon, \nu}^{2}+\varepsilon^{\frac{1+\alpha}{2}}\langle\xi\rangle_{\nu} E_{\varepsilon, \nu}^{2}+C_{0} \varepsilon^{-1} E_{\varepsilon, \nu}^{2} \\
& +(1+\alpha)\left|\left\{\left(-b+\varepsilon^{1+\alpha}\langle\xi\rangle_{\nu}^{2}\right)^{\frac{1}{1+\alpha}}\right\}^{\prime}\right| \varepsilon^{-1}\langle\xi\rangle_{\nu}^{-\frac{1}{2+\alpha}} E_{\varepsilon, \nu}^{2} \\
& +(1+\alpha)\left|\left\{\left(-b+\varepsilon^{1+\alpha}\langle\xi\rangle_{\nu}^{2}\right)^{\frac{1}{1+\alpha}}\right\}^{\prime}\right| \varepsilon^{-1}\langle\xi\rangle_{\nu}^{-\frac{2}{1+\alpha}} E_{\varepsilon, \nu}^{2} \\
& +2(1+\alpha)\left|\left\{\left(-b+\varepsilon^{1+\alpha}\langle\xi\rangle_{\nu}^{2}\right)^{\frac{1}{1+\alpha}}\right\}^{\prime}\right| \varepsilon^{-1}\langle\xi\rangle_{\nu}^{-\frac{2}{1+\alpha}} E_{\varepsilon, \nu}^{2} \\
& =\left(2 C_{0}+1\right) \varepsilon^{\frac{1+\alpha}{2}}\langle\xi\rangle_{\nu} E_{\varepsilon, \nu}^{2}+C_{0} \varepsilon^{-1} E_{\varepsilon, \nu}^{2}+4(1+\alpha)\left|\left\{\left(-b+\varepsilon^{1+\alpha}\langle\xi\rangle_{\nu}^{2}\right)^{\frac{1}{1+\alpha}}\right\}^{\prime}\right| \varepsilon^{-1}\langle\xi\rangle_{\nu}^{-\frac{2}{1+\alpha}} E_{\varepsilon, \nu}^{2}
\end{aligned}
$$

(39) $I I^{\prime}+I I I^{\prime}+I V^{\prime}$

$$
\begin{aligned}
& =2\left(a_{\varepsilon}-a\right) e^{\rho(t)\langle\xi\rangle} \varkappa_{\Re}\left(\frac{1}{2} a_{\varepsilon} v_{t}, v_{t t}+\frac{i}{2} a_{\varepsilon} v_{t}\right) \\
& +2 \varepsilon^{1+\alpha}\langle\xi\rangle_{\nu} e^{\rho(t)\langle\xi\rangle_{\nu}} \Re\left(\varepsilon^{\frac{1+\alpha}{4}}\langle\xi\rangle_{\nu}^{\frac{1}{2}} v_{t}, \varepsilon^{-\frac{1+\alpha}{4}}\langle\xi\rangle_{\nu}^{-\frac{1}{2}}\left(v_{t t}+\frac{i}{2} a_{\varepsilon} v_{t}\right)\right) \\
& +\frac{1}{2} a_{\varepsilon}^{\prime} \varepsilon^{-\frac{1+\alpha}{2}}\langle\xi\rangle_{\nu}^{-1} e^{\rho(t)\langle\xi\rangle_{\nu}^{x}} a_{\varepsilon} \varepsilon^{\frac{1+\alpha}{2}}\langle\xi\rangle_{\nu}\left|v_{t}\right|^{2}+\frac{-b^{\prime}}{-b+\varepsilon^{1+\alpha}\langle\xi\rangle_{\nu}^{2}} e^{\rho(t)\langle\xi\rangle_{\nu}^{x}}\left(-b+\varepsilon^{1+\alpha}\langle\xi\rangle_{\nu}^{2}\right)\left|v_{t}\right|^{2} \\
& +2 a_{\varepsilon}^{\prime} e^{\rho(t)\langle\xi\rangle} \Re \Re\left(\frac{i}{2} \varepsilon^{\frac{1+\alpha}{4}}\langle\xi\rangle_{\nu}^{\frac{1}{2}} v_{t}, \varepsilon^{-\frac{1+\alpha}{4}}\langle\xi\rangle_{\nu}^{-\frac{1}{2}}\left(v_{t t}+\frac{i}{2} a_{\varepsilon} v_{t}\right)\right) \\
& \leq C_{0} \varepsilon^{\frac{1+\alpha}{2}}\langle\xi\rangle_{\nu} E_{\varepsilon, \nu}^{2}+\varepsilon^{\frac{1+\alpha}{2}}\langle\xi\rangle_{\nu} E_{\varepsilon, \nu}^{2}+\frac{C_{0}}{2} \varepsilon^{-1} E_{\varepsilon, \nu}^{2} \\
& +(1+\alpha)\left|\left\{\left(-b+\varepsilon^{1+\alpha}\langle\xi\rangle_{\nu}^{2}\right)^{\frac{1}{1+\alpha}}\right\}^{\prime}\right| \varepsilon^{-1}\langle\xi\rangle_{\nu}^{-\frac{2}{1+\alpha}} E_{\varepsilon, \nu}^{2}+C_{0} \varepsilon^{-1} E_{\varepsilon, \nu}^{2}
\end{aligned}
$$




$$
=\left(C_{0}+1\right) \varepsilon^{\frac{1+\alpha}{2}}\langle\xi\rangle_{\nu} E_{\varepsilon, \nu}^{2}+\frac{3}{2} C_{0} \varepsilon^{-1} E_{\varepsilon, \nu}^{2}+(1+\alpha) \mid\left\{\left(-b+\varepsilon^{1+\alpha}\langle\xi\rangle_{\nu}^{2}\right)^{\left.\frac{1}{1+\alpha}\right\}^{\prime}} \mid \varepsilon^{-1}\langle\xi\rangle_{\nu}^{-\frac{2}{1+\alpha}} E_{\varepsilon, \nu}^{2} .\right.
$$

Therefore by (37), (38), (39) we have the estimate corresponding to (34) of the case of $k=0$

$$
\begin{aligned}
\frac{d}{d t}\left(E_{\varepsilon, \nu}^{2}\right) \leq & \rho^{\prime}(t)\langle\xi\rangle_{\nu}{ }_{\nu}^{\alpha} E_{\varepsilon, \nu}^{2}+C_{4} \varepsilon^{\frac{1+\alpha}{2}}\langle\xi\rangle_{\nu} E_{\varepsilon, \nu}^{2}+C_{5} \varepsilon^{-1} E_{\varepsilon, \nu}^{2} \\
& +C_{61}\left\{\left(-b+\varepsilon^{1+\alpha}\langle\xi\rangle_{\nu}^{2}\right)^{\frac{1}{1+\alpha}}\right\} \mid \varepsilon^{-1}\langle\xi\rangle_{\nu}^{-\frac{2}{1+\alpha}} E_{\varepsilon, \nu}^{2},
\end{aligned}
$$

where $C_{4}=3 C_{0}+1, C_{5}=\frac{5}{2} C_{0}, C_{6}=5(1+\alpha)$.

Thus Gronwall's inequality yields

$$
\begin{aligned}
& E_{\varepsilon, \nu}^{2}(t, \xi) \leq E_{\varepsilon, \nu}^{2}(0, \xi) \exp \left[\int _ { 0 } ^ { t } \left\{\rho^{\prime}(\tau)\langle\xi\rangle_{\nu}^{x}+C_{4} \varepsilon^{\frac{1+\alpha}{2}}\langle\xi\rangle_{\nu}+C_{5} \varepsilon^{-1}\right.\right. \\
& \left.\left.\quad+C_{6}\left|\left\{\left(-b(\tau)+\varepsilon^{1+\alpha}\langle\xi\rangle_{\nu}^{2}\right)^{\frac{1}{1+\alpha}}\right\}\right| \varepsilon^{-1}\langle\xi\rangle_{\nu}^{-\frac{2}{1+\alpha}}\right\}_{d} \tau\right] \text { for }{ }^{\forall} t \in[0, T] .
\end{aligned}
$$

Here we shall introduce the following useful lemma.

Lemmma. (Colombini, Jannelli, Spagnolo) Let $f(t)$ be a real function of class $C^{k+\alpha}$ on some compact interval $I \subset \mathbb{R}$, with $k$ integer $\geq 1$ and $0 \leq \alpha \leq 1$, and assume

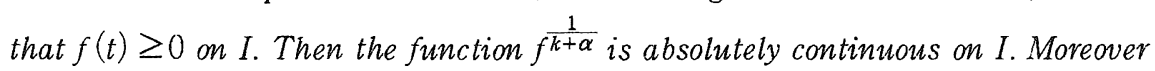

$$
\left\|\left(f^{\frac{1}{k+\alpha}}\right)^{\prime}\right\|_{L^{1}(I)}^{k+\alpha} \leq C(k, \alpha, I)\|f\|_{C^{i+\alpha}(I)} .
$$

For the proof, refer to [3]. From the condition (4) we can apply this lemma to $(40)$. Since $s\left(=\kappa^{-1}\right)<1+\frac{1+\alpha}{2}$, we find $\frac{2}{3+\alpha}-\kappa<0$ and $\langle\xi\rangle_{\nu}^{\frac{2}{3+\alpha}-\varkappa} \leq$ $\nu^{\frac{2}{3+\alpha}-x}$. Hence we take $\varepsilon=\langle\xi\rangle_{\nu}^{-\frac{2}{3+\alpha}}$ and obtain

$$
\begin{aligned}
E_{\varepsilon, \nu}^{2}(t, \xi) \leq & E_{\varepsilon, \nu}^{2}(0, \xi) \exp \left[\langle \xi \rangle _ { \nu } ^ { \varkappa } \int _ { 0 } ^ { t } \left\{\rho^{\prime}(\tau)+\left(C_{4}+C_{5}\right) \nu^{\frac{2}{3+\alpha}-\varkappa}\right.\right. \\
& \left.\left.+C_{6}\left|\left\{\left(-b(\tau)+\varepsilon^{1+\alpha}\langle\xi\rangle_{\nu}^{2}\right)^{\frac{1}{1+\alpha}}\right\}^{\prime}\right|\langle\xi\rangle_{\nu}^{-\frac{2}{1+\alpha}} \nu^{\frac{2}{3+\alpha}-\kappa}\right\} d \tau\right] \\
\leq & E_{\varepsilon, \nu}^{2}(0, \xi) \exp \left[\langle\xi\rangle_{\nu}^{\varkappa}\left\{\rho(t)-\rho_{0}+\left(C_{7} l+\phi_{1}(t)\right) \nu^{\frac{2}{3+\alpha}-\kappa}\right\}\right] \quad \text { for }{ }^{\forall} t \in[0, T],
\end{aligned}
$$

where $C_{7}=C_{4}+C_{5}, \phi_{1}(t)$ is a bounded function independent of $\xi$ and satisfy $\phi_{1}(0)=0$ and $\phi_{1}(t) \geq C_{6} C(t)\left\|-b+\varepsilon^{1+\alpha}\langle\xi\rangle_{\nu}^{2}\right\|_{C^{1+\alpha}}^{\frac{1}{1+\alpha}}\langle\xi\rangle_{\nu}^{-\frac{2}{1+\alpha}}$.

Moreover we determine $\rho(t)=\rho_{0}-\left(C_{7} t+\phi_{1}(t)\right) \frac{2}{\nu^{3+\alpha}-x}$. and choose $\nu>0$ such that $\rho(T)\left(=\rho_{0}-\left(C_{7} T+\phi_{1}(T)\right) \nu^{\frac{2}{3+\alpha}-x}\right)>0$ for any given $T>0$.

Finally we have the energy inequality

$$
E_{\varepsilon, \nu}^{2}(t, \xi) \leq E_{\varepsilon, \nu}^{2}(0, \xi) \quad \text { for }{ }^{\forall} t \in[0, T] \text { and }{ }^{\forall} \xi \in \mathbb{R}_{\xi}^{n} .
$$




\subsection{Case of $k \geq 2$}

We finally treat the case of $k \geq 2$ which implies that both coefficients $a(t, \xi), b(t, \xi)$ belong to $C^{1}$ class in $t$ at least. Therefore we don't need to regularize the coefficients $a(t, \xi), b(t, \xi)$.

With the coefficients $a(t, \xi), b(t, \xi)$ we shall define the following energy.

$$
\begin{aligned}
E_{\varepsilon, \nu}(t, \xi)^{2}=e^{\rho(t)\langle\xi\rangle_{\nu}}\left\{\left|v_{t t}+i a(t, \xi) v_{t}-b(t, \xi) v+\varepsilon^{k+\alpha}\langle\xi\rangle_{\nu} \nu\right|^{2}\right. \\
\left.+\left|v_{t t}+\frac{i}{2} a(t, \xi) v_{t}\right|^{2}+\left(\frac{a(t, \xi)^{2}}{4}-b(t, \xi)+\varepsilon^{k+\alpha}\langle\xi\rangle_{\nu}^{2}\right)\left|v_{t}\right|^{2}\right\} .
\end{aligned}
$$

Differentiating (42) in $t$, by (23) we get

$$
\begin{aligned}
\frac{d}{d t}\left(E_{\varepsilon, \nu}^{2}\right)= & \rho^{\prime}(t)\langle\xi\rangle_{\nu}{ }_{\nu}^{2} E_{\varepsilon, \nu}^{2} \\
& +2 e^{\rho(t)\langle\xi\rangle\rangle_{\nu}^{x}} \Re\left(\varepsilon^{k+\alpha}\langle\xi\rangle_{\nu}^{2} v_{t}+i a^{\prime} v_{t}-b^{\prime} v_{,} v_{t t}+i a v_{t}-b v+\varepsilon^{k+\alpha}\langle\xi\rangle_{\nu} 2 v\right) \\
& +2 e^{\rho(t)\left\langle\xi \xi{ }_{\nu}^{x}\right.} \Re\left(-\frac{i}{2} a v_{t t}+b v_{t}+\frac{i}{2} a^{\prime} v_{t}, v_{t t}+\frac{i}{2} a v_{t}\right) \\
& +2\left(\frac{a^{2}}{4}-b+\varepsilon^{k+\alpha}\langle\xi\rangle_{\nu}^{2}\right) e^{\rho(t)\langle\xi\rangle_{\nu}^{x}} \Re\left(v_{t}, v_{t t}\right)+e^{\rho(t)\langle\xi\rangle}\left(\frac{1}{2} a a^{\prime}-b^{\prime}\right)\left|v_{t}\right|^{2} \\
( & \left.\equiv \rho^{\prime}(t)\langle\xi\rangle_{\nu}{ }_{\nu} E_{\varepsilon, \nu}^{2}+I^{\prime \prime}+I I^{\prime \prime}+I I I^{\prime \prime}+I V^{\prime \prime}\right)
\end{aligned}
$$

Before we estimate the terms $I^{\prime \prime}$ and $I I^{\prime \prime}+I I I^{\prime \prime}+I V^{\prime \prime}$, we shall culculate the parts concerned with the coefficient $a(t)$ in advance. From the condition $|a(t)| \in$ $C^{\frac{k+\alpha}{2}}([0, T])\left(\subset C^{1}([0, T])\right)$, we can see $\left|a^{\prime}(t)\right|=\|\left. a(t)\right|^{\prime} \mid$. Hence it holds that for $w(t, \xi)=v_{t t}+i a(t, \xi) v_{t}-b(t, \xi) v+\varepsilon^{k+\alpha}\langle\xi\rangle_{\nu}^{2} v$ or $v_{t t}+\frac{i}{2} a(t, \xi) v_{t}$

(44) $2 a^{\prime} e^{\rho(t)\langle\xi\rangle \nu} \Re\left(i\left(\frac{|a|}{2}+\varepsilon^{\frac{k+\alpha}{2}}\langle\xi\rangle_{\nu}\right)^{\frac{1}{2}} v_{t},\left(\frac{|a|}{2}+\varepsilon^{\frac{k+\alpha}{2}}\langle\xi\rangle_{\nu}\right)^{-\frac{1}{2}} w\right)$

$$
\begin{aligned}
& \leq\left|a^{\prime}\right| e^{\rho(t)\langle\xi\rangle_{\nu} x}\left\{\left(\frac{|a|}{2}+\varepsilon^{\frac{k+\alpha}{2}}\langle\xi\rangle_{\nu}\right)\left|v_{t}\right|^{2}+\left(\frac{|a|^{2}}{2}+\varepsilon^{\frac{k+\alpha}{2}}\langle\xi\rangle_{\nu}\right)^{-1}|w|^{2}\right\} \\
& =\frac{\left|\left\{\left(\frac{|a|}{2}+\varepsilon^{\frac{k+\alpha}{2}}\langle\xi\rangle_{\nu}\right)^{2}\right\}\right|}{\frac{a^{2}}{4}+\varepsilon^{k+\alpha}\langle\xi\rangle_{\nu}^{2}} e^{\rho(t)\langle\xi\rangle_{\nu}}\left(\frac{a^{2}}{4}+\varepsilon^{k+\alpha}\langle\xi\rangle_{\nu}\right)\left|v_{t}\right|^{2}+2 \frac{\left|\left(\frac{|a|}{2}+\varepsilon^{\frac{k+\alpha}{2}}\langle\xi\rangle_{\nu}\right)^{\prime}\right|}{\frac{|a|}{2}+\varepsilon^{\frac{k+\alpha}{2}}\langle\xi\rangle_{\nu}} e^{\rho(t)\langle\xi)^{\prime} \mid}|w|^{2} \\
& \leq 2 \frac{\left|\left\{\left(\frac{|a|}{2}+\varepsilon^{\frac{k+\alpha}{2}}\langle\xi\rangle_{\nu}\right)^{2}\right\}^{\prime}\right|}{\left(\frac{|a|}{2}+\varepsilon^{\frac{k+\alpha}{2}}\langle\xi\rangle_{\nu}\right)^{2}} E_{\varepsilon, \nu}^{2}+2 \frac{\left|\left(\frac{|a|}{2}+\varepsilon^{\frac{k+\alpha}{2}}\langle\xi\rangle_{\nu}\right)^{\prime}\right|}{\frac{|a|}{2}+\varepsilon^{\frac{k+\alpha}{2}}\langle\xi\rangle_{\nu}} E_{\varepsilon, \nu}^{2} \\
& \leq 3(k+\alpha)\left|\left\{\left(\frac{|a|}{2}+\varepsilon^{\frac{k+\alpha}{2}}\langle\xi\rangle_{\nu}\right)^{\frac{2}{k+\alpha}}\right\}\right| \varepsilon^{-1}\langle\xi\rangle_{\nu}^{-\frac{2}{k+\alpha}} E_{\varepsilon, \nu .}^{2} .
\end{aligned}
$$


And it also holds that

$$
\begin{aligned}
a a^{\prime} e^{\left.\rho(t)\langle\xi\rangle^{\prime}\right\rangle}\left|v_{t}\right|^{2} & \leq \frac{2\left(\frac{|a|}{2}+\varepsilon^{\frac{k+\alpha}{2}}\langle\xi\rangle_{\nu}\right) \cdot 2\left|\left(\frac{|a|}{2}+\varepsilon^{\frac{k+\alpha}{2}}\langle\xi\rangle_{\nu}\right)^{\prime}\right|}{\frac{a^{2}}{4}+\varepsilon^{k+\alpha}\langle\xi\rangle_{\nu}^{2}} e^{\rho(t)\langle\xi\rangle^{\prime} \nu}\left(\frac{a^{2}}{4}+\varepsilon^{k+\alpha}\langle\xi\rangle_{\nu}\right)\left|v_{t}\right|^{2} \\
& \leq 4 \frac{\left|\left\{\left(\frac{|a|}{2}+\varepsilon^{\frac{k+\alpha}{2}}\langle\xi\rangle_{\nu}\right)^{2}\right\}^{\prime}\right|}{\left(\frac{|a|}{2}+\varepsilon^{\frac{k+\alpha}{2}}\langle\xi\rangle_{\nu}\right)^{2}} E_{\varepsilon, \nu}^{2} \\
& \leq 4(k+\alpha)\left|\left\{\left(\frac{|a|}{2}+\varepsilon^{\frac{k+\alpha}{2}}\langle\xi\rangle_{\nu}\right)^{\frac{2}{k+\alpha}}\right\}^{\prime}\right| \varepsilon^{-1}\langle\xi\rangle_{\nu}^{-\frac{2}{k+\alpha}} E_{\varepsilon, \nu}^{2} .
\end{aligned}
$$

In (44), (45) we used $\frac{a^{2}}{4}+\varepsilon^{k+\alpha}\langle\xi\rangle_{\nu}^{2} \geq \frac{1}{2}\left(\frac{|a|}{2}+\varepsilon^{\frac{k+\alpha}{2}}\langle\xi\rangle_{\nu}\right)^{2}$.

Noting that $\frac{-b^{\prime}}{-b+\varepsilon^{k+\alpha}\langle\xi\rangle_{\nu}^{2}} \leq(k+\alpha)\left|\left\{\left(-b+\varepsilon^{k+\alpha}\langle\xi\rangle_{\nu}^{2}\right)^{\frac{1}{k+\alpha}}\right\}^{\prime}\right| \varepsilon^{-1}\langle\xi\rangle_{\nu}^{-\frac{2}{k+\alpha}}$, and using (44) with $w(t, \xi)=v_{t t}+i a(t, \xi) v_{t}-b(t, \xi) v+\varepsilon^{k+\alpha}\langle\xi\rangle{ }_{2}^{2} v$, similarly as other cases, we can estimate $I^{\prime \prime}$ as follows.

$$
\begin{aligned}
& \text { (46) } I^{\prime \prime}=2 \varepsilon^{k+\alpha}\langle\xi\rangle_{L^{2}}^{2} e^{(t)\langle\xi\rangle} \cdot \Re\left(\varepsilon^{\frac{k+\alpha}{4}}\langle\xi\rangle_{\nu}^{\frac{1}{2}} v_{t}, \varepsilon^{-\frac{k+\alpha}{4}}\langle\xi\rangle_{\nu}^{-\frac{1}{2}}\left(v_{t t}+i a v_{t}-b v+\varepsilon^{k+\alpha}\langle\xi\rangle_{\nu}^{2} v\right)\right) \\
& +2 a^{\prime} e^{\rho(t)\langle\xi\rangle_{\nu}} \Re\left(i\left(\frac{|a|}{2}+\varepsilon^{\frac{k+\alpha}{2}}\langle\xi\rangle_{\nu}\right)^{\frac{1}{2}} v_{t},\right. \\
& \left.\left(\frac{|a|}{2}+\varepsilon^{\frac{k+\alpha}{2}}\langle\xi\rangle_{\nu}\right)^{-\frac{1}{2}}\left(v_{t t}+i a v_{t}-b v+\varepsilon^{k+\alpha}\langle\xi\rangle_{\nu}^{2} v\right)\right) \\
& +2 \frac{-b^{\prime}}{-b+\varepsilon^{k+\alpha}\langle\xi\rangle_{\nu}^{2}} e^{\left.\rho(t){ }^{\prime} \xi\right\rangle_{\nu}^{*}} \Re\left(-v_{t t}-\frac{i}{2} a v_{t}, v_{t t}+i a v_{t}-b v+\varepsilon^{k+\alpha}\langle\xi\rangle_{\nu}^{2} v\right) \\
& +2 \frac{-b^{\prime}}{-b+\varepsilon^{k+\alpha}\langle\xi\rangle_{\nu}^{2}} e^{\rho(t)\left\langle\xi{ }^{k}\right.} \Re\left(-\frac{i}{2} a v_{t}, v_{t t}+i a v_{t}-b v+\varepsilon^{k+\alpha}\langle\xi\rangle_{\nu}^{2} v\right)
\end{aligned}
$$

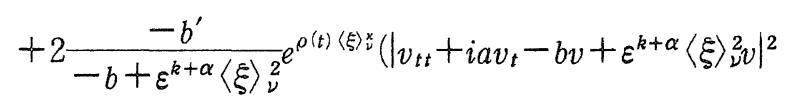

$$
\begin{aligned}
& \leq \varepsilon^{\frac{k+\alpha}{2}}\langle\xi\rangle_{\nu} E_{\varepsilon, \nu}^{2}+3(k+\alpha)\left|\left\{\left(\frac{|a|}{2}+\varepsilon^{\frac{k+\alpha}{2}}\langle\xi\rangle_{\nu}\right)^{\frac{2}{k+\alpha}}\right\}^{\prime}\right| \varepsilon^{-1}\langle\xi\rangle_{\nu}^{-\frac{2}{k+\alpha}} E_{\varepsilon, \nu}^{2} \\
& +(k+\alpha)\left|\left\{\left(-b+\varepsilon^{k+\alpha}\langle\xi\rangle_{\nu}^{2}\right)^{\frac{1}{k+\alpha}}\right\}^{\prime}\right| \varepsilon^{-1}\langle\xi\rangle_{\nu}^{-\frac{2}{k+\alpha}} E_{\varepsilon, \nu}^{2} \\
& +(k+\alpha)\left|\left\{\left(-b+\varepsilon^{k+\alpha}\langle\xi\rangle_{\nu}^{2}\right)^{\frac{1}{k+\alpha}}\right\}^{\prime}\right| \varepsilon^{-1}\langle\xi\rangle_{\nu}^{-\frac{2}{k+\alpha}} E_{\varepsilon, \nu}^{2} \\
& +2(k+\alpha)\left|\left\{\left(-b+\varepsilon^{k+\alpha}\langle\xi\rangle_{\nu}^{2}\right)^{\frac{1}{k+\alpha}}\right\}^{\prime}\right| \varepsilon^{-1}\langle\xi\rangle_{\nu}^{-\frac{2}{k+\alpha}} E_{\varepsilon, \nu}^{2} \\
& =\varepsilon^{\frac{k+\alpha}{2}}\langle\xi\rangle_{\nu} E_{\varepsilon, \nu}^{2}+3(k+\alpha)\left|\left\{\left(\frac{|a|}{2}+\varepsilon^{\frac{k+\alpha}{2}}\langle\xi\rangle_{\nu}\right)^{\frac{2}{k+\alpha}}\right\}\right| \varepsilon^{-1}\langle\xi\rangle_{\nu}^{-\frac{2}{k+\alpha}} E_{\varepsilon, \nu}^{2} \\
& +4(k+\alpha)\left|\left\{\left(-b+\varepsilon^{k+\alpha}\langle\xi\rangle_{\nu}^{2}\right)^{\frac{1}{k+\alpha}}\right\}^{\prime}\right| \varepsilon^{-1}\langle\xi\rangle_{\nu}^{-\frac{2}{k+\alpha}} E_{\varepsilon, \nu}^{2}
\end{aligned}
$$


Aa for $I I^{\prime \prime}+I I I^{\prime \prime}+I V^{\prime \prime}$, using (44) $w(t, \xi)$ with $v_{t t}+\frac{i}{2} a(t, \xi) v_{t}$ and (45), similarly as other cases, we can get

$$
\begin{aligned}
& I I^{\prime \prime}+I I I^{\prime \prime}+I V=2 \varepsilon^{k+\alpha}\langle\xi\rangle_{\nu}^{2} e^{\rho(t)\langle\xi\rangle_{\nu}} \Re\left(\varepsilon^{\frac{k+\alpha}{4}}\langle\xi\rangle_{\nu}^{\frac{1}{2}} v_{t}, \varepsilon^{-\frac{k+\alpha}{4}}\langle\xi\rangle_{\nu}^{-\frac{1}{2}}\left(v_{t t}+\frac{i}{2} a v_{t}\right)\right) \\
& +\frac{1}{2} a a^{\prime} e^{\rho(t)\langle\xi\rangle}\left|v_{t}\right|^{2}+\frac{-b^{\prime}}{-b+\varepsilon^{k+\alpha}\langle\xi\rangle_{\nu}} e^{\rho(t)\langle\xi\rangle_{\nu}^{k}}\left(-b+\varepsilon^{k+\alpha}\langle\xi\rangle_{\nu}^{2}\right)\left|v_{t}\right|^{2} \\
& +a^{\prime} e^{\left.\rho(t)^{\prime}{ }^{\prime}\right\rangle^{\prime} x_{\mathfrak{R}}}\left(i\left(\frac{|a|}{2}+\varepsilon^{\frac{k+\alpha}{2}}\langle\xi\rangle_{\nu}\right)^{\frac{1}{2}} v_{t},\left(\frac{|a|}{2}+\frac{k+\alpha}{2}\langle\xi\rangle_{\nu}\right)^{-\frac{1}{2}}\left(v_{t t}+\frac{i}{2} a v_{t}\right)\right) \\
& \leq \varepsilon^{\frac{k+\alpha}{2}}\langle\xi\rangle_{\nu} E_{\varepsilon, \nu}^{2}+2(k+\alpha)\left|\left\{\left(\frac{|a|}{2}+\varepsilon^{\frac{k+\alpha}{2}}\langle\xi\rangle_{\nu}\right)^{\frac{2}{k+\alpha}}\right\}\right| \varepsilon^{-1}\langle\xi\rangle_{\nu}^{-\frac{2}{k+\alpha}} E_{\varepsilon, \nu}^{2} \\
& +(k+\alpha)\left|\left\{\left(-b+\varepsilon^{k+\alpha}\langle\xi\rangle_{\nu}^{2}\right)^{\frac{1}{k+\alpha}}\right\}^{\prime}\right| \varepsilon^{-1}\langle\xi\rangle_{\nu}^{-\frac{2}{k+\alpha}} E_{\varepsilon, \nu}^{2} \\
& +\frac{3}{2}(k+\alpha)\left|\left\{\left(\frac{|a|}{2}+\varepsilon^{\frac{k+\alpha}{2}}\langle\xi\rangle_{\nu}\right)^{\frac{2}{k+\alpha}}\right\}^{\prime}\right| \varepsilon^{-1}\langle\xi\rangle_{\nu}^{-\frac{2}{k+\alpha}} E_{\varepsilon, \nu}^{2} \\
& =\varepsilon^{\frac{k+\alpha}{2}}\langle\xi\rangle_{\nu} E_{\varepsilon, \nu}^{2}+(k+\alpha)\left|\left\{\left(-b+\varepsilon^{k+\alpha}\langle\xi\rangle_{\nu}^{2}\right)^{\frac{1}{k+\alpha}}\right\}^{\prime}\right| \varepsilon^{-1}\langle\xi\rangle_{\nu}^{-\frac{2}{k+\alpha}} E_{\varepsilon, \nu}^{2} \\
& +\frac{7}{2}(k+\alpha)\left|\left\{\left(\frac{|a|}{2}+\varepsilon^{\frac{k+\alpha}{2}}\langle\xi\rangle_{\nu}\right)^{\frac{2}{k+\alpha}}\right\}^{\prime}\right| \varepsilon^{-1}\langle\xi\rangle_{\nu}^{-\frac{2}{k+\alpha}} E_{\varepsilon, \nu}^{2} \text {. }
\end{aligned}
$$

Therefore by $(43),(46),(47)$ we have the estimate

$$
\begin{gathered}
\frac{d}{d t}\left(E_{\varepsilon, \nu}^{2}\right) \leq \rho^{\prime}(t)\langle\xi\rangle_{\nu} E_{\varepsilon, \nu}^{2}+C_{8} \varepsilon^{\frac{k+\alpha}{2}}\langle\xi\rangle_{\nu} E_{\varepsilon, \nu}^{2}+C_{9}\left|\left\{\left(-b+\varepsilon^{k+\alpha}\langle\xi\rangle_{\nu}^{2}\right)^{\frac{1}{k+\alpha}}\right\}^{\prime}\right| \varepsilon^{-1}\langle\xi\rangle_{\nu}^{-\frac{2}{k+\alpha}} E_{\varepsilon, \nu}^{2} \\
+C_{10}\left|\left\{\left(\frac{|a|}{2}+\varepsilon^{\frac{k+\alpha}{2}}\langle\xi\rangle_{\nu}\right)^{\frac{2}{k+\alpha}}\right\}\right| \varepsilon^{-1}\langle\xi\rangle_{\nu}^{-\frac{2}{k+\alpha}} E_{\varepsilon, \nu}^{2},
\end{gathered}
$$

where $C_{8}=2, C_{9}=5(k+\alpha), C_{10}=\frac{13}{3}(k+\alpha)$.

Thus Gronwall's inequality yields

$$
\begin{aligned}
E_{\varepsilon, \nu}^{2}(t, \xi) & \leq E_{\varepsilon, \nu}^{2}(0, \xi) \exp \left[\int _ { 0 } ^ { t } \left\{\rho^{\prime}(\tau)\langle\xi\rangle_{\nu}{ }_{\nu}+C_{8} \varepsilon^{\frac{k+\alpha}{2}}\langle\xi\rangle_{\nu}+C_{9}\left|\left\{\left(-b+\varepsilon^{k+\alpha}\langle\xi\rangle_{\nu}^{2}\right)^{\frac{1}{k+\alpha}}\right\}^{\prime}\right| \varepsilon^{-1}\langle\xi\rangle_{\nu}^{-\frac{2}{k+\alpha}}\right.\right. \\
& \left.\left.+C_{10}\left|\left\{\left(\frac{|a|}{2}+\varepsilon^{\frac{k+\alpha}{2}}\langle\xi\rangle_{\nu}\right)^{\frac{2}{k+\alpha}}\right\}\right| \varepsilon^{-1}\langle\xi\rangle_{\nu} \frac{2}{k+\alpha}\right\} d \tau\right] \quad \text { for } \quad{ }^{\forall} t \in[0, T] .
\end{aligned}
$$

Hence we shall also apply the lemma in $\mathbf{2 . 2}$ to this inequality. Noting that $s\left(=\kappa^{-1}\right)<1+\frac{k+\alpha}{2}\left(\frac{2}{2+k+\alpha}-\kappa<0\right)$, and taking $\varepsilon=\langle\xi\rangle_{\nu}^{-\frac{2}{2+k+\alpha}}$, we obtain

$$
\begin{aligned}
& E_{\varepsilon, \nu}^{2}(t, \xi) \leq E_{\varepsilon, \nu}^{2}(0, \xi) \\
& \times \exp \left[\langle \xi \rangle _ { \nu } ^ { x } \int _ { 0 } ^ { t } \left\{\rho^{\prime}(\tau)+C_{8} \nu^{\frac{2}{2+k+\alpha}-\kappa}+C_{9}\left|\left\{\left(-b(\tau)+\varepsilon^{k+\alpha}\langle\xi\rangle_{\nu}^{2}\right)^{\frac{1}{k+\alpha}}\right\}^{\prime}\right|\langle\xi\rangle_{\nu}^{-\frac{2}{k+\alpha}} \nu^{\frac{2}{2+k+\alpha}-\kappa}\right.\right. \\
& \left.\left.+C_{10}\left|\left\{\left(\frac{|a(\tau)|}{2}+\varepsilon^{\frac{k+\alpha}{2}}\langle\xi\rangle_{\nu}\right)^{\frac{2}{k+\alpha}}\right\}\right|\langle\xi\rangle_{\nu}^{-\frac{2}{k+\alpha}} \nu^{\frac{2}{2+k+\alpha}-x}\right\} d \tau\right] \\
& =E_{\varepsilon, \nu}^{2}(0, \xi) \exp \left[\langle\xi\rangle_{\nu}\left\{\rho(t)-\rho_{0}+\left(C_{8} t+\phi_{2}(t)\right) \nu^{\frac{2}{2+k+\alpha}}-x\right\}\right] \text { for }{ }^{\forall} t \in[0, T] \text {, }
\end{aligned}
$$


where $\phi_{2}(t)$ is a bounded function independent of $\xi$ and satisfies $\phi_{2}(0)=0$ and

$$
\phi_{2}(t) \geq C_{9} C(t)\left\|-b+\varepsilon^{k+\alpha}\langle\xi\rangle_{\nu}^{2}\right\|_{C^{k+\alpha}}^{\frac{1}{k+\alpha}}\langle\xi\rangle_{\nu}^{-\frac{2}{k+\alpha}}+C_{10} \widetilde{C}(t)\left\|\frac{|a|}{2}+\varepsilon^{\frac{k+\alpha}{2}}\langle\xi\rangle_{\nu}\right\|_{C^{k+\alpha}}^{\frac{2}{k+\alpha}}\langle\xi\rangle_{\nu}^{-\frac{2}{k+\alpha}} .
$$

Similarly as other cases we determine $\rho(t)=\rho_{0}-\left(C_{8} t+\phi_{2}(t)\right) \nu^{\frac{2}{2+k+\alpha}}-\varkappa$, and choose $\nu>0$ such that $\rho(T)\left(=\rho_{0}-\left(C_{8} T+\phi_{2}(T)\right) \nu^{\frac{2}{2+k+\alpha}-x}\right)>0$ for any given $T>0$.

Finally we have the energy inequality

$$
E_{\varepsilon, \nu}^{2}(t, \xi) \leq E_{\varepsilon, \nu}^{2}(0, \xi) \quad \text { for } \quad{ }^{\forall} t \in[0, T] \text { and }{ }^{\forall} \xi \in \mathbb{R}_{\xi}^{n} .
$$

\subsection{Conclusion of the preoof}

Putting

$$
a_{(\varepsilon)}(t, \xi)=\left\{\begin{array}{lc}
a_{\varepsilon}(t, \xi) & \text { for } k=0,1 \\
a(t, \xi) & \text { for } k=2,3 \cdots,
\end{array} \quad b_{(\varepsilon)}(t, \xi)= \begin{cases}b_{\varepsilon}(t, \xi) & \text { for } k=0 \\
b(t, \xi) & \text { for } k=1,2, \cdots\end{cases}\right.
$$

we can alter the definitions of energies (28). (36), (42) into the following.

$$
\begin{aligned}
E_{\varepsilon, \nu}(t, \xi)^{2}= & e^{\rho\left(t,\left\langle\xi^{\xi}\right\rangle \nu\right.}\left\{\left|v_{t t}+i a_{(\varepsilon)}(t, \xi) v_{t}-b_{(\varepsilon)}(t, \xi) v+\varepsilon^{k+\alpha}\langle\xi\rangle_{\nu}^{2} v\right|^{2}\right. \\
& \left.+\left|v_{t t}+\frac{i}{2} a_{(\varepsilon)}(t, \xi) v_{t}\right|^{2}+\left(\frac{a_{(\varepsilon)}(t, \xi)^{2}}{4}-b_{(\varepsilon)}(t, \xi)+\varepsilon^{k+\alpha}\langle\xi\rangle_{\nu}^{2}\right)\left|v_{t}\right|^{2}\right\} .
\end{aligned}
$$

Since the energy inequalities (35). (41), (48) are same, it holds that

$$
E_{\varepsilon, \nu}^{2}(t, \xi) \leq E_{\varepsilon, \nu}^{2}(0, \xi) \text { for }{ }^{\forall} t \in[0, T] \text { and }{ }^{\vee} \xi \in \mathbb{R}_{\xi}^{n} \text {. }
$$

We shall change the energy inequality (51) into the inequality based on the absolute values of $v, v_{t}$ and $v_{t t}$. For this aim we must investigate the relations between the energy $(50)$ and the absolute values of $v, v_{t}$ and $v_{t t}$.

From the definition of energy (50) we can easily see

$$
E_{\varepsilon, \nu}^{2} \geq e^{\rho(t)\langle\bar{\xi} \times} \times\left(\frac{a_{(\varepsilon)}(t, \xi)^{2}}{4}-b_{(\varepsilon)}(t, \xi)+\varepsilon^{k+\alpha}\langle\xi\rangle_{\nu}^{2}\right)\left|v_{t}\right|^{2} \geq e^{\rho(t)\langle\xi)_{\nu}^{*}}\langle\xi\rangle_{\nu}^{\frac{4}{2+k+\alpha}}\left|v_{t}\right|^{2}
$$

We can also find

$$
\begin{aligned}
E_{\varepsilon, \nu}^{2} & \geq e^{\rho(t)\langle\xi\rangle \nu}\left\{\left|v_{t t}+\frac{i}{2} a_{(\varepsilon)} v_{t}\right|^{2}+\frac{a_{(\varepsilon)}^{2}}{4}\left|v_{t}\right|^{2}\right\} \\
& =e^{\rho(t)\langle\xi\rangle x}\left\{\frac{1}{2}\left|v_{t t}\right|^{2}+\frac{1}{2}\left|v_{t t}\right|^{2}+\Re\left(v_{t t}, i a_{(\varepsilon)} v_{t}\right)+\frac{a_{(\varepsilon)}^{2}}{4}\left|v_{t}\right|^{2}\right\} \\
& =e^{\rho(t)\langle\langle\xi\rangle \Sigma}\left\{\frac{1}{2}\left|v_{t t}\right|^{2}+\frac{1}{2}\left|v_{t t}+i a_{(\varepsilon)} v_{t}\right|^{2}\right\} \\
& \geq \frac{1}{2} e^{\rho(t)\langle\xi) \downarrow}\left|v_{t t}\right|^{2} .
\end{aligned}
$$


Moreover we can see

$$
\begin{aligned}
& E_{\varepsilon, \nu}^{2} \geq e^{\rho(t)\langle\xi\rangle^{k}}\left\{\left|\left(v_{t t}+\frac{i}{2} a_{(\varepsilon)} v_{t}\right)+\left(\frac{i}{2} a_{(\varepsilon)} v_{t}-b_{(\varepsilon)} v+\varepsilon^{k+a}\langle\xi\rangle{ }_{\nu}^{2} v\right)\right|^{2}\right. \\
& \left.+\left|v_{t t}+\frac{i}{2} a_{(\varepsilon)} v_{t}\right|^{2}+\frac{a_{(\varepsilon)}^{2}}{4}\left|v_{t}\right|^{2}\right\} \\
& =\left.e^{\rho(t)\langle\xi\rangle \nu}\left\{\mid \sqrt{2}\left(v_{t t}+\frac{i}{2} a_{(\varepsilon)} v_{t}\right)+\frac{1}{\sqrt{2}}\left(\frac{i}{2} a_{(\varepsilon)} v_{t}-b_{(\varepsilon)} v+\varepsilon^{k+\alpha}\langle\xi\rangle\right\rangle_{\nu} v\right)\right|^{2} \\
& \left.+\left.\frac{1}{2}\left|\frac{i}{2} a_{(\varepsilon)} v_{t}-b_{(\varepsilon) v}+\varepsilon^{k+\alpha}\langle\xi\rangle\right\rangle_{\nu}^{2}\right|^{2}+\frac{a_{(\varepsilon)}^{2}}{4}\left|v_{t}\right|^{2}\right\} \\
& \geq e^{\rho(t)\langle\xi\rangle \nu}\left\{\frac{1}{2}\left|\frac{i}{2} a_{(\varepsilon) v_{t}}-b_{(\varepsilon) v}+\varepsilon^{k+\alpha}\langle\xi\rangle_{\nu}^{2} v\right|^{2}+\frac{a_{(\varepsilon)}^{2}}{4}\left|v_{t}\right|^{2}\right\} \\
& =e^{\rho(t)\langle\xi\rangle}\left\{\frac{1}{2}\left|\frac{\sqrt{3} i}{2} a_{(\varepsilon)} v_{t}+\frac{1}{\sqrt{3}}\left(-b_{(\varepsilon)} v+\varepsilon^{k+\alpha}\langle\xi\rangle_{\nu}^{2} v\right)\right|^{2}+\frac{1}{3}\left|-b_{(\varepsilon)} v+\varepsilon^{k+\alpha}\langle\xi\rangle_{\nu} \nu\right|^{2}\right\} \\
& \geq e^{\rho(t)\langle\xi)_{\nu}} \frac{1}{3}\left(-b_{(\varepsilon)}+\varepsilon^{k+\alpha}\langle\xi\rangle_{\nu}^{2}\right)^{2}|v|^{2} \\
& \geq \frac{1}{3} e^{\rho(t)\langle\xi\rangle_{\nu}} \varepsilon^{2(k+\alpha)}\langle\xi\rangle_{\nu}^{4}|v|^{2}=\frac{1}{3} e^{\left.\rho^{\prime} t\right\rangle\langle\xi\rangle_{\nu}^{*}}\langle\xi\rangle_{\nu}{ }_{\nu}^{\frac{8}{2+k+\alpha}}|v|^{2} .
\end{aligned}
$$

While the energy $E_{\varepsilon, \nu}(0, \xi)$ can be also dominated by the absolute values of the initial data. From the definitions of the energy (50) we get

$$
\begin{aligned}
& E_{\varepsilon, \nu}(0)^{2}=e^{\rho_{0}\langle\xi\rangle \nu}\left\{\left.\left|v_{2}+i a_{(\varepsilon)}(0) v_{1}-b_{(\varepsilon)}(0) v_{0}+\varepsilon^{k+\alpha}\langle\xi\rangle\right\rangle_{\nu}^{2} v_{0}\right|^{2}\right. \\
& \left.+\left|v_{2}+\frac{i}{2} a_{(\varepsilon)}(0) v_{1}\right|^{2}+\left(\frac{a_{(\varepsilon)}(0)^{2}}{4}-b_{(\varepsilon)}(0)+\varepsilon^{k+\alpha}\langle\xi\rangle_{\nu}^{2}\right)\left|v_{1}\right|^{2}\right\} \\
& =e^{\rho_{0}(\xi){ }_{\nu}}\left\{2\left|v_{2}\right|^{2}+\left(\frac{3}{2} a_{(\varepsilon)}(0)^{2}-b_{(\varepsilon)}(0)+\varepsilon^{k+a}\langle\xi\rangle_{\nu}\right)\left|v_{1}\right|^{2}+2 \Re\left(v_{2}, \frac{3 i}{2} a_{(\varepsilon)}(0) v_{1}\right)\right. \\
& +\left(-b_{(\varepsilon)}(0)+\varepsilon^{k+\alpha}\langle\xi\rangle_{\nu}^{2}\right)^{2}\left|v_{0}\right|^{2}+2 \Re\left(v_{2},\left(-b_{(\varepsilon)}(0)+\varepsilon^{k+\alpha}\langle\xi\rangle_{\nu}^{2}\right) v_{0}\right) \\
& \left.+2 \Re\left(i a_{(\varepsilon)}(0) v_{1},\left(-b_{(\varepsilon)}(0)+\varepsilon^{k+\alpha}\langle\xi\rangle_{\nu}^{2}\right) v_{0}\right)\right\} \\
& \leq e^{\rho_{0}\langle\xi\rangle_{\nu} \times}\left\{4\left|v_{2}\right|^{2}+\left(\frac{19}{4} a_{(\varepsilon)}(0)^{2}-b_{(\varepsilon)}(0)+\varepsilon^{k+\alpha}\langle\xi\rangle_{\nu}^{2}\right)\left|{\iota^{\prime}}_{1}\right|^{2}\right. \\
& \left.+3\left(-b_{(\varepsilon)}(0)+\varepsilon^{k+\alpha}\langle\xi\rangle_{\nu}^{2}\right)^{2}\left|v_{0}\right|^{2}\right\} \\
& \leq C e^{\left.\rho_{0}(\xi)\right\rangle}\left(\langle\xi\rangle_{\nu}^{4}\left|v_{0}\right|^{2}+\langle\xi\rangle_{\nu}^{2}\left|v_{1}\right|^{2}+\left|v_{2}\right|^{2}\right) \text {. }
\end{aligned}
$$

Using the energy inequalities (51), by (22) and (52) - (55) we have the energy inequality based on the absolute values of $v, v_{t}$ and $v_{t t}$

$$
\begin{aligned}
e^{\rho(t)\langle\xi)_{\nu}}\left(\langle\xi\rangle_{\nu}^{\frac{8}{2+k+\alpha}}|v|^{2}+\right. & \left.\langle\xi\rangle_{\nu}^{\frac{4}{2+k+\alpha}}\left|v_{t}\right|^{2}+\left|v_{t t}\right|^{2}\right) \\
& \leq{ }^{\exists} C_{a b} e^{\rho_{0}\langle\xi\rangle_{\nu}^{*}}\left(\langle\xi\rangle_{\nu}^{4}\left|v_{0}\right|^{2}+\langle\xi\rangle_{\nu}^{2}\left|v_{1}\right|^{2}+\left|v_{2}\right|^{2}\right)(<\infty) .
\end{aligned}
$$


and by square root of the both sides we also have

$$
\begin{aligned}
e^{\frac{\rho(t)}{2}\langle\xi\rangle_{\nu}}\left(\langle\xi\rangle_{\nu}^{\frac{4}{2+k+\alpha}}|v|+\langle\xi\rangle_{\nu}^{\frac{2}{2+k+\alpha}} \mid\right. & \left.v_{t}|+| v_{t t} \mid\right) \\
& \leq{ }^{\exists} C_{a b} e^{\frac{\rho_{0}}{2}\langle\xi\rangle_{\nu}}\left(\langle\xi\rangle_{\nu}^{2}\left|v_{0}\right|+\langle\xi\rangle_{\nu}\left|v_{1}\right|+\left|v_{2}\right|\right)(<\infty) .
\end{aligned}
$$

Putting $\mu(t)=\frac{1}{2} \rho(t), \mu_{0}=\frac{1}{2} \rho_{0}$, we can see that (56) implies (7). In vitue of Paley-Wiener theorem, $\{u(\cdot, t) ; t \in[0, T]\}$ is bounded in $\gamma_{0}^{s}$. Thus taking into account that $u$ is a solution of $(1)$, we find $u \in C^{3}\left([0, T], \gamma_{0}^{s}\right)$. This concludes the proof of Theorem 1 .

\section{§ 3. Proof of Theorem 2}

We shall only introduce the definition of the energy.

$$
\begin{aligned}
E_{\varepsilon, \nu}(t, \xi)^{2}= & e^{\rho(t)\langle\xi\rangle \nu}\left\{\left|v_{t t}+i a_{\varepsilon}(t, \xi) v_{t}-b_{\varepsilon}(t, \xi) v\right|^{2}\right. \\
& \left.+\left|v_{t t}+\frac{i}{2} a_{\varepsilon}(t, \xi) v_{t}\right|^{2}+\left(\frac{a_{\varepsilon}(t, \xi)^{2}}{4}-b_{\varepsilon}(t, \xi)\right)\left|v_{t}\right|^{2}\right\} .
\end{aligned}
$$

We remark that for this energy there exists the constant $C>0$ such that $C^{-1} e^{\rho(t)\langle\xi\rangle_{\nu}^{x}}\left(\langle\xi\rangle_{\nu}^{4}|v|^{2}+\langle\xi\rangle_{\nu}^{2}\left|v_{t}\right|^{2}+\left|v_{t t}\right|^{2} \leq E_{\varepsilon, \nu}(t, \xi)^{2} \leq C e^{\rho_{0}\langle\xi\rangle_{\nu}^{x}}\left(\langle\xi\rangle_{\nu}^{4}\left|v_{0}\right|^{2}+\langle\xi\rangle_{\nu}^{2}\left|v_{1}\right|^{2}+\left|v_{2}\right|^{2}\right)\right.$

Similarly if $s\left(=\kappa^{-1}\right)$ satisfies (10), we can get the energy inequality

$$
e^{\frac{\rho(t)^{2}\langle\xi\rangle_{\nu}^{*}}{2}}\left(\langle\xi\rangle_{\nu}^{2}|v|+\langle\xi\rangle_{\nu}\left|v_{t}\right|+\left|v_{t t}\right|\right) \leq{ }^{\exists} C_{a b}^{\prime} e^{\frac{\rho_{0}\langle\xi\rangle_{\nu}^{*}}{2}}\left(\left.\left.\langle\xi\rangle_{\nu}\right|_{\nu} v_{0}\right|^{2}+\langle\xi\rangle_{\nu}\left|v_{1}\right|+\left|v_{2}\right|\right)(<\infty) .
$$

Putting $\mu(t)=\frac{1}{2} \rho(t), \mu_{0}=\frac{1}{2} \rho_{0}$, we can see that (58) implies (11).

\section{§4. Proof of Corollary 3}

In this section our task is also to derive the energy inequality. By Fourier transform the Cauthy problem (12) is changed to

$$
\left\{\begin{array}{l}
\mathscr{F}[L u](t, \xi)=-c(t, \xi) v+i d(t, \xi) v_{t}+e(t) v_{t t}+i f(t, \xi) v+g(t) v_{t}+h(t) v \\
v(0, \xi)=v_{0}(\xi), \quad v_{t}(0, \xi)=v_{1}(x), \quad v_{t t}(0, \xi)=v_{2}(\xi)
\end{array}\right.
$$

where $c(t, \xi)=\sum_{i, j=1}^{n} c_{i j}(t) \xi_{i} \xi_{j}, d(t, \xi)=\sum_{i=1}^{n} d_{i}(t) \xi_{i}, f(t, \xi)=\sum_{i=1}^{n} f_{i}(t) \xi_{i}$.

In order to reduce the proof to the argument of $\S 2$, we shall culculate the parts concerned with the lower terms.

In (29), (37), (43) the following term also appears. 


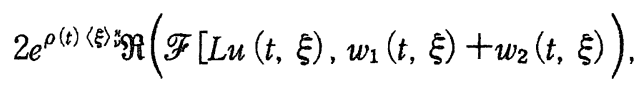

where $w_{1}(t, \xi)=v_{t t}+i a_{(\varepsilon)} v_{t}-b_{(\varepsilon)} v+\varepsilon^{k+\alpha}\langle\xi\rangle_{\nu}^{2} v, w_{2}(t, \xi)=v_{t t}+\frac{i}{2} a_{(\varepsilon)} v_{t}$.

Picking up the each term in $\mathscr{F}[L u](t, \xi)$, we shall estimate.

i) the lower term $-c(t, \xi) v$

Noting that (54), from the assumption (14) we get for $l=1,2$

$$
\begin{aligned}
& 2 e^{\rho(t)\langle\xi\rangle^{x}} \Re\left(-c(t, \xi) v, w_{l}(t, \xi)\right) \\
& \leq\left. 2 e^{\rho(t)\langle\xi\rangle}\right|_{c}(t, \xi) v \| w_{l}\left|\leq 2 e^{\rho(t)\langle\xi\rangle_{\nu}^{*}} \sigma_{1}\left(-b_{(\varepsilon)}+\varepsilon^{k+\alpha}\langle\xi\rangle_{\nu}^{2}\right)^{\beta_{1}}\right| v|| w_{l} \mid \\
& \leq e^{\rho(t)\langle\xi\rangle_{\nu}^{x}} \sigma_{1}\left(-b_{(\varepsilon)}+\varepsilon^{k+\alpha}\langle\xi\rangle_{\nu}^{2}\right)^{\beta_{1}-1}\left\{\left(-b_{(\varepsilon)}+\varepsilon^{k+\alpha}\langle\xi\rangle_{\nu}^{2}\right)^{2}|v|^{2}+\left|w_{l}\right|^{2}\right\} \\
& \leq 4 e^{\rho(t)\langle\xi\rangle_{\nu}^{x}} \sigma_{1}\left(-b_{(\varepsilon)}+\varepsilon^{k+\alpha}\langle\xi\rangle_{\nu}^{2}\right)^{\beta_{1}-1} E_{\varepsilon, \nu}^{2} \\
& \leq 4 e^{\rho(t)\langle\xi\rangle x}\left(\sigma_{1}\langle\xi\rangle^{2 \beta_{1}-2}\right) \varepsilon^{(k+\alpha)\left(\beta_{1}-1\right)} E_{\varepsilon, \nu}^{2} \text {. }
\end{aligned}
$$

Hence we obtain

$$
2 e^{\rho(t)\langle\xi\rangle_{n}} \mathfrak{R}\left(-c(t, \xi) v, w_{1}\left((t, \xi)+w_{2}(t, \xi)\right) \leq C_{11} e^{\rho(t)\langle\xi\rangle_{\nu}^{x}}\left(\sigma_{1}\langle\xi\rangle^{2 \beta_{1}-2}\right) \varepsilon^{(k+\alpha)\left(\beta_{1}-1\right)} E_{\varepsilon, \nu}^{2}\right.
$$

where $C_{11}=8$.

ii) the lower term $i d(t, \xi) v_{t}$

Noting that (52), from the assumption (15) we get for $l=1,2$

$$
\begin{aligned}
& 2 e^{\left.\rho^{\prime(t)\langle\xi\rangle}\right\rangle_{\nu}} \Re\left(i d(t, \xi) v_{t}, w_{l}(t, \xi)\right) \\
& \leq 2 e^{\rho(t)\langle\xi\rangle_{\nu}^{k}}\left|d(t, \xi) v\left\|w_{l}\left|\leq 2 e^{\rho(i)\langle\xi\rangle_{\nu}^{x}} \sigma_{2}\left(a_{(\varepsilon)}^{2}-4 b_{(\varepsilon)}+4 \varepsilon^{k+\alpha}\langle\xi\rangle_{\nu}^{2}\right)^{\beta_{2}}\right| v_{t}\right\| w_{l}\right| \\
& \leq 4^{\beta 2} e^{\rho(t)\langle\xi\rangle_{\nu}{ }^{k}} \sigma_{2}\left(\frac{a_{(\varepsilon)}^{2}}{4}-b_{(\varepsilon)}+\varepsilon^{k+\alpha}\langle\xi\rangle_{\nu}^{2}\right)^{\beta_{2}-\frac{1}{2}}\left\{\left(\frac{a_{(\varepsilon)}^{2}}{4}-b_{(\varepsilon)}+\varepsilon^{k+\alpha}\langle\xi\rangle_{\nu}^{2}\right)\left|v_{t}\right|^{2}+\left|w_{l}\right|^{2}\right\} \\
& \leq 2^{2 \beta_{2}+1} e^{\rho(t)\langle\xi\rangle\rangle} \sigma_{2}\left(\frac{a_{(\varepsilon)}^{2}}{4}-b_{(\varepsilon)}+\varepsilon^{k+\alpha}\langle\xi\rangle_{\nu}^{2}\right)^{\beta_{2}-\frac{1}{2}} E_{\varepsilon, \nu}^{2} \\
& \leq 2^{2 \beta_{2}+1} e^{\rho(t)\langle\xi\rangle \nu}\left(\sigma_{2}\langle\xi\rangle^{2 \beta_{2}-1}\right) \varepsilon^{(k+\alpha)\left(\beta_{2}-\frac{1}{2}\right)} E_{\varepsilon, \nu}^{2}
\end{aligned}
$$

Hence we obtain

(62) $2 e^{\rho(t)\langle\xi\rangle\rangle^{*}} \Re\left(i d(t, \xi) v, w_{1}(t, \xi)+w_{2}(t, \xi)\right) \leq C_{12} e^{\rho(t)\langle\xi \xi \nu}\left(\sigma_{2}\langle\xi\rangle^{2 \beta_{2}-1}\right) \varepsilon^{(k+\alpha)\left(\beta_{1}-\frac{1}{2}\right)} E_{\varepsilon, \nu,}^{2}$, where $C_{12}=2^{2 \beta 2+2}$.

iii) the lower term $e(t) v_{t t}$ 
Noting that (53), we get for $l=1,2$

$$
\begin{aligned}
& 2 e^{\rho(t)\langle\xi\rangle} \Re\left(e(t) v_{t t}, w_{l}(t, \xi)\right) \leq 2 e^{\rho(t)\left\langle\xi{ }^{x}\right.}\left|e(t) v_{t t}\right|\left|w_{l}\right| \\
& \leq e^{\rho(t)\langle\xi\rangle^{\prime} v}|e(t)|\left(\left|v_{t t}\right|^{2}+\left|w_{l}\right|^{2}\right) \leq 2 e^{\rho(t)\langle\xi\rangle^{\prime}}|e(t)| E_{\varepsilon, \nu}^{2} .
\end{aligned}
$$

Hence we obtain

$$
2 e^{\rho(t)\langle\xi\rangle} \kappa_{\Re} R\left(e(t) v_{t t}, w_{1}(t, \xi)+w_{2}(t, \xi)\right) \leq C_{13} 2 e^{\rho(t)\langle\xi\rangle}|e(t)| E_{\varepsilon, \nu}^{2},
$$

where $C_{13}=4$.

iv) the lower term if $(t, \xi) v$

Noting that (54), similarly as i) from the assumption (16) we obtain (64) $2 e^{\rho(t)\langle\xi\rangle\rangle} \Re\left(i f(t, \xi) v, w_{1}(t, \xi)+w_{2}(t, \xi)\right) \leq C_{14} e^{\rho(t)\langle\xi\rangle_{\nu}}\left(\sigma_{3}\langle\xi\rangle^{2 \beta_{3}-1}\right)\langle\xi\rangle_{\nu}^{-1} \varepsilon^{(k+\alpha)\left(\beta_{3}-1\right)} E_{\varepsilon, \nu}^{2}$ where $C_{14}=8$.

v) the lower term $g(t) v_{t}$

Noting that (52), we get for $l=1,2$

$$
\begin{aligned}
& 2 e^{\rho(t)\langle\xi\rangle} \stackrel{\Re}{R}\left(g(t) v_{t}, w_{l}(t, \xi)\right) \leq 2 e^{\rho(t)\langle\xi\rangle}\left|g(t) v_{t} \| w_{l}\right| \\
& \leq e^{\rho(t)\langle\xi\rangle}|g(t)| \varepsilon^{-\frac{k+\alpha}{2}}\langle\xi\rangle_{\nu}^{-1}\left(\varepsilon^{k+\alpha}\langle\xi\rangle_{\nu}\left|v_{t}\right|^{2}+\left|w_{l}\right|^{2}\right) \leq 2 e^{\rho(t)\langle\xi\rangle}|g(t)| \varepsilon^{-\frac{k+\alpha}{2}}\langle\xi\rangle_{\nu}^{-1} E_{\varepsilon, \nu}^{2} .
\end{aligned}
$$

Hence we obtain

$$
2 e^{\rho(t)\langle\xi\rangle} \Re \Re\left(g(t) v_{t}, w_{1}(t, \xi)+w_{2}(t, \xi)\right) \leq C_{15} e^{\rho(t)\left\langle\xi \xi^{\prime} \nu\right.}|g(t)| \varepsilon^{-\frac{k+\alpha}{2}}\langle\xi\rangle_{\nu}^{-1} E_{\varepsilon, \nu}^{2}
$$

where $C_{15}=4$.

vi) the lower term $h(t) v$

Noting that (54), we obtain

$$
2 e^{\rho(t)\langle\xi\rangle} \stackrel{x}{R}\left(h(t) v, w_{1}(t, \xi)+w_{2}(t, \xi)\right) \leq C_{16} e^{\rho(t)\langle\xi\rangle \nu}|h(t)| \varepsilon^{-(k+\alpha)}\langle\xi\rangle{ }_{\nu}^{2} E_{\varepsilon, \nu}^{2}
$$

where $C_{16}=8$.

Having (13), (17), (18), (19) and (59) - (66) under consideration, with the similar methods as the proof of Theorem 1 , we take

$$
\left.\varepsilon=\langle\xi\rangle_{\nu}{ }^{-\min \left\{\frac{2}{2+k+\alpha}\right.}, \frac{1}{(k+\alpha)\left(\frac{3}{2}-\beta_{1}\right)}, \frac{1}{(k+\alpha)\left(1-\beta_{2}\right)} \cdot \frac{2}{(k+\alpha)\left(\frac{3}{2}-\beta_{3}\right)}\right\}
$$

and determine 


$$
\rho(t)=\rho_{0}-\left(C_{17} t+\phi_{3}(t)\right) \nu^{\max \left\{\frac{2}{2+k+\alpha}, \frac{1-\beta_{1}}{\frac{3}{2}-\beta_{1}}, \frac{\frac{1}{2}-\beta_{2}}{1-\beta_{2}}, \frac{1}{2}-\frac{\beta_{3}}{3}-\beta_{3}\right\}-\kappa},
$$

and choose $\nu\left(\geq \nu_{0}\right)$ such that

$$
\rho(T)=\rho_{0}-\left(C_{17} T+\phi_{3}(T)\right) \nu^{\max \left\{\frac{2}{2+k+\alpha}, \frac{1-\beta_{1}}{\frac{3}{2}-\beta_{1}}, \frac{\frac{1}{2}-\beta_{2}}{1-\beta_{2}}, \frac{\frac{1}{2}-\beta_{3}}{\frac{3}{2}-\beta_{3}}\right\}-\kappa}>0
$$

for any given $T>0$.

In conclusion we also get the energy inequality (51) if the lower terms satisfy (13) which is the condition concerned with regularity and (14). (15), (16) which are the conditions corresponding to a sort of Oleinik condition for the third order equation (12) and $s\left(=\kappa^{-1}\right)$ satisfies (19).

Moreover noting that $E_{\varepsilon, \nu}^{2} \geq e^{\rho(t)\langle\xi\rangle_{\nu}^{x}}\langle\xi\rangle_{\nu}^{2 \omega}\left|v_{t}\right|^{2}, E_{\varepsilon, \nu}^{2} \geq \frac{1}{3} e^{\rho(t)\langle\xi\rangle}\langle\xi \xi\rangle_{\nu}^{4 \omega}|v|^{2}$, we also have the energy inequality based on the absolute values of $v, v_{t}$ and $v_{t t}$

$$
\begin{aligned}
e^{\frac{\rho(t)}{2}\langle\xi\rangle_{\nu}^{*}}\left(\langle\xi\rangle_{\nu}^{2 \omega}|v|\right. & \left.+\langle\xi\rangle_{\nu}^{\omega}\left|v_{t}\right|+\left|v_{t t}\right|\right) \\
& \leq{ }^{\exists} C_{a b} e^{\frac{\rho_{0}}{2}\langle\xi\rangle_{\nu}^{*}}\left(\langle\xi\rangle_{\nu}^{2}\left|v_{0}\right|+\langle\xi\rangle_{\nu}\left|v_{1}\right|+\left|v_{2}\right|\right)(<\infty),
\end{aligned}
$$

where $\omega$ is the constant defined in (21).

Putting $\mu(t)=\frac{1}{2} \rho(t), \mu_{0}=\frac{1}{2} \rho_{0}$, we can see that (67) implies (20).

\section{References}

[1] Cicognanı, M., On the strictly hyperbolic equations which are Hölder continuous with respect to time, Preprint.

[2] Colombini, F., De Giorg1, E. and Spagnolo, S., Sur les équations hyperboliques avec des coefficients qui ne dépendent que du temps, Ann. Scuola Norm Sup. Pisa, 6 (1979), 511-559.

[3] Colombini, F., Jannelli, E. and Spagnolo, S., Wellposedness in the Gevrey classes of the Cauchy problem for a non strictly hyperbolic equation with coefficients depending on time. Ann. Scuola Norm Sup. Pisa, 10 (1983), 291-312.

[4] D'ancona, P., Gevrey well posedness of an abstract Cauchy problem of weakly hyperbolic type, Publ. RIMS, Kyoto Univ.. 24 (1988), 433-449.

[5] Ivrii, V., Cauchy problem conditions for hyperbolic operators with characteristics of variable multiplicity for Gevrey classes, Siberian. Math., 17 (1976), 921-931.

[6] Jannelli, E.. Gevrey well-posedness for a class of weakly hyperbolic equations, J. Math. Kyoto Univ., 24-4 (1984), 763-778.

[7] Linear Kovalevskıan systems with time dependent coefficients. Comm. P. D. E., 9 (1984), 1373-1406.

[8] Regularly hyperbolic systems and Gevrey classes, Ann. Mat. Pura Appl., CXL (1985), 133-145. 
[9] Kajitani, K., The well posed Cauchy problem for hyperbolic operators, Exposé au Séminaire de Vaillant du 8 février, (1989).

[10] Nishitani, T., Sur les équations hyperboliues à coefficients hölderiens en $t$ et de classes de Gevrey en $x$, Bull. Sci. Math., 107 (1983), 739-773.

[11] Ohya, Y. and Tarama, S., Le problème de Cauchy à caractéristiques multiplescoefficients hölderiens en $t^{-}$, Proc. Tanıguchi Intern. Sympos. on Hyperbolic Equations and Related Topics 1984, 273-306.

[12] Ovciannikov, V., Singular operators in Banach scales, Dokl. Akad. Nauk. SSSR., 163 (1965), 819-822 (Soviet. Math. Dokl., 6 (1965), 1025-1028).

[13] Reissing, M. and Yagdjian, K., Levi conditions and global Gevrey regularity for the solutions of quasilinear weakly hyperbolic equations, Math. Nachr., 178 (1996), 285-307.

[14] Tarama, S., On the initial value problem for the weakly hyperbolic operators in the Gevrey classes, (Proc. of the conference on hyperbolic equations, university of Pisa, 1987), Longman Scientific and Technical, 1988, 322-339. 